\title{
Eddy interaction model for turbulent suspension in Reynolds-averaged Euler-Lagrange simulations of steady sheet flow
}

\author{
Zhen Cheng ${ }^{\mathrm{a}, \mathrm{b}, *}$, Julien Chauchat ${ }^{\mathrm{c}}$, Tian-Jian $\mathrm{Hsu}^{\mathrm{a}}$, Joseph Calantoni ${ }^{\mathrm{d}}$ \\ ${ }^{a}$ Civil and Environmental Engineering, University of Delaware, Newark, DE 19716, U.S.A. \\ ${ }^{b}$ Now at Applied Ocean Physics $\&$ Engineering, Woods Hole Oceanographic Institution, Woods Hole, \\ MA 02543, USA \\ ${ }^{c}$ Laboratory of Geophysical and Industrial Flows (LEGI), BP 53, 38041 Grenoble Cedex 9, FR \\ ${ }^{d}$ Marine Geosciences Division, U.S. Naval Research Laboratory, Stennis Space Center, MS 39529, USA
}

\begin{abstract}
A Reynolds-averaged Euler-Lagrange sediment transport model (CFDEM-EIM) was developed for steady sheet flow, where the inter-granular interactions were resolved and the flow turbulence was modeled with a low Reynolds number corrected $k-\omega$ turbulence closure modified for two-phase flows. To model the effect of turbulence on the sediment suspension, the interaction between the turbulent eddies and particles was simulated with an eddy interaction model (EIM). The EIM was first calibrated with measurements from dilute suspension experiments. We demonstrated that the eddy-interaction model was able to reproduce the well-known Rouse profile for suspended sediment concentration. The model results were found to be sensitive to the choice of the coefficient, $C_{0}$, associated with the turbulence-sediment interaction time. A value $C_{0}=3$ was suggested to match the measured concentration in the dilute suspension. The calibrated CFDEMEIM was used to model a steady sheet flow experiment of lightweight coarse particles and yielded reasonable agreements with measured velocity, concentration and turbulence kinetic energy profiles. Further numerical experiments for sheet flow suggested that when $C_{0}$ was decreased to $C_{0}<3$, the simulation under-predicted the amount of suspended sediment in the dilute region and the Schmidt number is over-predicted $(S c>1.0)$. Ad-
\end{abstract}

\footnotetext{
${ }^{*}$ Corresponding author

Email address: zcheng@whoi.edu (Zhen Cheng)
} 
ditional simulations for a range of Shields parameters between 0.3 and 1.2 confirmed that CFDEM-EIM was capable of predicting sediment transport rates similar to empirical formulations. Based on the analysis of sediment transport rate and transport layer thickness, the EIM and the resulting suspended load were shown to be important when the fall parameter is less than 1.25 .

Keywords: Euler-Lagrange model, eddy interaction model, turbulent suspension, steady sheet flow, Rouse profile, sediment transport rate

\section{1. Introduction}

Studying sediment transport in rivers and coastal regions is critical to understand the fluvial geomorphology, loss of wetland, and beach erosion. For example, significant engineering efforts were devoted to control the river discharge and sediment budget to reduce the loss of Louisiana wetland (Mossa, 1996; Allison et al., 2012). In the Indian River inlet, significant erosion of the north beach is mitigated through proper beach nourishment that interacts with littoral drift (Keshtpoor et al., 2013). The characteristics of sediment transport vary significantly with sediment properties and flow conditions, and it is widely believed that sheet flow plays a dominant role in nearshore beach erosion and riverine sediment delivery, especially during storm and flood conditions, respectively.

Sheet flow is an intense sediment transport mode, in which a thick layer of concentrated sediment is mobilized above the quasi-static bed. The conventional single-phasebased sediment transport models assume the dynamics of transport can be subjectively separated into bedload and suspended load (e.g., van Rijn, 1984a b). While the suspended load are directly resolved, the bedload are parameterized by empirical formulations. Several laboratory measurements of sheet flow with the full profile of sediment transport flux and net transport rate indicated that the split of bedload and suspended load may be too simple because sediment entrainment/deposition is a continuous and highly dynamic process near the mobile bed (e.g., O’Donoghue and Wright, 2004; Revil-Baudard et al. 2015). In sheet flow, the two prevailing mechanisms driving the sediment transport are inter-granular interactions and turbulent suspension (Revil-Baudard et al., 2015, Jenkins 
and Hanes, 1998). In order to model the full profile of sediment transport, both mechanisms must be taken into account. In the past decade, many Eulerian two-phase flow models have been developed for sheet flow transport in steady (Jenkins and Hanes, 1998; Longo, 2005; Revil-Baudard and Chauchat, 2013) and oscillatory flows (Dong and Zhang, 2002; Hsu et al., 2004; Amoudry et al., 2008; Liu and Sato, 2006; Chen et al., 2011; Cheng et al. 2017a). By solving the mass and momentum equations of fluid phase and sediment phase with appropriate closures for interphase momentum transfer, turbulence, and intergranular stresses, these models are able to resolve the entire profiles of sediment transport without the assumption of bedload and suspended load.

In the continuum description of the sediment phase, the assumption of uniform particle properties and spherical particle shapes are usually adopted. To better capture the polydisperse nature of sediment transport and irregular particle shapes, the Lagrangian approach for the particle phase, namely the Discrete Element Method (DEM, Cundall and Strack, 1979; Maurin et al., 2015; Sun and Xiao, 2016a) is superior to the Eulerian approach because individual particle properties may be uniquely specified (Calantoni et al. 2004; Harada and Gotoh, 2008; Fukuoka et al., 2014). One of the main challenges in modeling sheet flow arise from the various length scales involved in inter-granular interactions and sediment-turbulence interactions. To resolve the flow turbulence and turbulencesediment interactions in sheet flow, the computational domain needs to be sufficiently large to resolve the largest eddies, while the grid resolution should be small enough to resolve the energy containing turbulent eddies. This constrain becomes even more challenging in the Euler-Lagrange modeling framework. Large domains require both a large number of grid points to resolve a sufficient amount of turbulence energy cascade (i.e., large-eddy simulation) and a large number of particles in a given simulation (e.g., Finn et al., 2016). It is well-established that in sheet flow, the transport layer thickness scales with the grain size and the Shields parameter (Wilson, 1987), suggesting that a common sheet flow layer thickness must be about several tens of grain diameters. To simulate the largest eddies and their subsequent cascade, the domain lengths in the two horizontal directions must be proportional to the boundary layer thickness, which is usually about 
several tens of centimeters. For a bed layer thickness of 50 grains with a typical grain diameter of $0.2 \mathrm{~mm}$, sheet flow simulations may require at least several tens of millions of particles. Therefore, to efficiently model sediment transport for many scenarios in sheet flow, a turbulence-averaged approach for the carrier phase may be necessary. In a turbulence-averaged formulation, turbulent eddies are not resolved and their effects on the averaged flow field are often parameterized via eddy viscosity. In this case, the domain lengths in the two horizontal directions are solely determined by the largest length scale of inter-granular interaction, which is usually captured within 50 grain diameters (Maurin et al. 2015). Consequently, the number of particles needed for each sheet flow simulation is limited to no more than a few hundred thousand.

With a goal to develop a robust open-source coupled Computational Fluid Dynamics Discrete Element Method (CFD-DEM) for sheet flow applications, we adopt a turbulenceaveraged approach in this study. Existing Reynolds-averaged CFD-DEM models have the capability to model bedload transport (Durán et al., 2012; Maurin et al., 2015) and sheet flow for coarse sand (Drake and Calantoni, 2001), where the inter-granular interactions are dominant, and the turbulent suspension is of minor importance. The previous studies made significant progresses in understanding the sediment dynamics due to intergranular collisions and interactions with the mean flow, and the key characteristics such as sediment transport rate and transport layer thickness close to the empirical formulations were obtained. In more energetic sheet flows with medium to fine sand particles, the role of turbulence-induced suspension can become important, where substantial sediment suspension occurs above the bedload layer (Bagnold, 1966; Sumer et al., 1996). In such condition, a more complete closure models for turbulent suspension and turbulence modulation by particles are needed. The natural way of describing the diffusion and dispersion of dispersed particles is to sample the turbulent velocity statistics along their trajectories in a stochastic manner (Taylor, 1922), and this idea lays the foundation of modeling the turbulent motions of particles with a Lagrangian approach.

In the stochastic Lagrange model for particle dispersion, the turbulent agitation to the sediment particles are considered either through a random-walk model (RWM) or an 
eddy interaction model (EIM). In the RWM framework, the strength of particle velocity fluctuations are typically assumed to be similar to the fluid turbulence, and a series of random velocity fluctuations are directly added to the particle velocities. While the Lagrange model with RWM is successful in studying the particle dispersion in mixing layer (Coimbra et al., 1998) and dilute suspension (Shi and Yu, 2015), the assumption of estimating the particle velocity fluctuations based on the fluid turbulence is crucial, and many researchers found that the correlation between the particle and fluid fluctuations are highly dependent on the particle Stokes number, $S t=t_{p} / t_{l}$ (Balachandar and Eaton 2010), where $t_{p}$ is the particle response time, and $t_{l}$ is the characteristic time scale of energetic eddies. For the particles with very small inertia $(S t \ll 1)$, they can closely follow the eddy motion. However, if $S t \gg 1$, the particle trajectory is hardly affected by the fluid eddy motion. Due to the particle inertia effect, it was found that the fluid turbulent intensity needs to be enhanced for medium to coarse particles (Shi and Yu, 2015). This problem can be largely remedied by the EIM (Matida et al., 2004), where the fluid velocity fluctuations associated with the fluid turbulence are added through the particle-sediment interaction force, i.e., the drag force. This approach incorporates the particle inertia effect naturally and it is applicable for a wide range of sediment properties. Graham (1996) found that the dispersion of inertial particles may be correctly represented with a suitable choice of maximum interaction time and length scales with the eddies. This model was later improved by using a randomly sampled eddy interaction time, in which more realistic turbulent scales become possible, and the enhanced dispersion of high-inertia particles are captured. In the previous studies of particle dispersion (e.g., Shi and $\mathrm{Yu}, 2015)$, the turbulent intensity is either prescribed from the empirical formula, or modeled using clear fluid turbulence closure without considering turbulence modulation by the presence of particles. In sheet flow, it is well-known that the sediment-turbulence interaction is important in attenuating the flow turbulence, thus the presence of sediment can dissipate/enhance flow turbulence through drag/density stratification.

In this paper, we present an application of the eddy interaction model (EIM) in a Reynolds-averaged Euler-Lagrange formulation to study sheet flow. The eddy interaction 
model is implemented into an open source coupled CFD-DEM scheme called CFDEM (Goniva et al., 2012), and the new solver is called CFDEM-EIM. The fluid phase is modeled in a similar way as the Eulerian two-phase flow model SedFOAM (Cheng et al. 2017a), and the particles are modeled with the discrete particle model, LIGGGHTS (Kloss et al., 2012). The paper is organized in the following manner. The model formulation is described in Section 2. The model calibration with dilute suspension experiments is presented in Section 3.1. followed by model validation of steady sheet flow (Section 3.2) using a comprehensive dataset (Revil-Baudard et al., 2015, 2016). Section 4 discusses the model sensitivity of the resulting sediment diffusivity and Schmidt number to model coefficients in the eddy interaction scheme, and effects of the EIM on the modeled sediment transport rate and transport layer thickness are also evaluated. Finally, a practical regime for the EIM to be important is proposed based on the fall parameter. Concluding remarks are given in Section 5

\section{Model formulations}

\subsection{Discrete particle model}

In the framework of the discrete element method (Cundall and Strack, 1979), the position of each particle is tracked by integrating the particle equation of motion,

$$
\frac{d \mathbf{x}_{p, i}}{d t}=\mathbf{v}_{i}
$$

where $\mathbf{x}_{p, i}$ is the position of particle $i$ and $\mathbf{v}_{i}$ is the translational velocity. The governing equation for the translational motion of particle $i$ with radius $r_{i}$ and mass $m_{i}$ may be written as,

$$
m_{i} \frac{d \mathbf{v}_{i}}{d t}=\mathbf{f}_{p f, i}+\sum_{j=1}^{N_{c}}\left(\mathbf{f}_{n, i j}+\mathbf{f}_{t, i j}\right)+m_{i} \mathbf{g} .
$$

The forces acting on the $i$-th particle include the particle-fluid interaction force, $\mathbf{f}_{p f, i}$, the gravitational force, $m_{i} \mathbf{g}$, and the normal, $\mathbf{f}_{n, i j}$, and tangential, $\mathbf{f}_{t, i j}$, contact forces where $N_{c}$ is the number of particles in contact with the particle $i$. The rotational motion of 
particle $i$ with moment of inertia $I_{i}$ may be written as,

$$
I_{i} \frac{d \boldsymbol{\Omega}_{i}}{d t}=\sum_{j=1}^{N_{c}}\left(\mathbf{M}_{t, i j}+\mathbf{M}_{r, i j}\right),
$$

where $\Omega_{i}$ is the angular velocity of particle $i$. The torque acting on particle $i$ from particle $j$ consists of two components. Closures are used for $\mathbf{M}_{t, i j}$, which is generated by the tangential force, and $\mathbf{M}_{r, i j}$, which is commonly known as the rolling friction torque (Luding, 2008).

To model grain contact forces, we adopt the soft-sphere approach Cundall and Strack, 1979) based on Hertz-Mindlin theory. Hertz theory is implemented in the normal direction, and the improved Mindlin no-slip model is implemented in the shear direction (Mindlin, 1949). In the soft-sphere model (e.g., Di Renzo and Di Maio, 2005), particles are allowed to overlap slightly, and the contact between two particles may be described as a nonlinear spring-dashpot, where the normal contact force, $\mathbf{f}_{n, i j}$, is determined by the overlap, $\delta_{i j}$, and relative velocity between colliding particles, $\mathbf{V}_{\mathbf{r}, \mathbf{i j}}$, while the tangental force, $\mathbf{f}_{t, i j}$, is calculated in a similar way and includes the tangental contact history. In addition, if the tangential force exceeds the Coulomb frictional limit, the particles begin to slide, and the tangential force is set to $\mathbf{f}_{t, i j}=\mu_{c} \mathbf{f}_{n, i j}$, where $\mu_{c}$ is the Coulomb friction coefficient. In the present study, we only consider the torque induced by particle-particle/particle-wall contact, and the influence of fluid-induced torque is ignored.

In general, the particle-fluid interaction force, $\mathbf{f}_{p f}$, is the sum of all types of particlefluid interaction forces on individual particles by fluid, including the so-called drag force, $\mathbf{f}_{d}$, the pressure gradient force, $f_{p}$, buoyancy force if assuming locally hydrostatic flow, virtual mass force, $\mathbf{f}_{v m}$, Basset force, $\mathbf{f}_{B}$ and lift forces such as the Saffman force, $\mathbf{f}_{S a f f}$, and the Magnus force, $\mathbf{f}_{\text {Mag }}$. We assume that the fluid and particles share the pressure field, thus the fluid pressure gradient force is also included in the fluid-particle interactions (Maxey and Riley, 1983; Zhou et al., 2010). In CFDEM-EIM, only the two dominant forces, namely the drag force and pressure gradient force, are retained. Here the total fluid-particle interaction force acting on particle $i$ may be written as,

$$
\mathbf{f}_{p f, i}=\mathbf{f}_{d, i}+\mathbf{f}_{p, i}
$$


The pressure gradient force acting on particle $i$ is calculated as,

$$
\mathbf{f}_{p, i}=\left(\mathbf{f}_{x, i}-\nabla_{i} p\right) \cdot V_{i}
$$

where $\mathbf{f}_{x, i}$ is the external body force driving the steady flow. $\nabla_{i} p$ is the interpolated fluid pressure gradient at particle $i$, and $V_{i}$ is the volume of particle $i$. The drag force acting on particle $i$ is expressed as,

$$
\mathbf{f}_{d, i}=\frac{1}{2} C_{D} A_{s, i}\left|\mathbf{u}_{f, i}-\mathbf{v}_{i}\right|\left(\mathbf{u}_{f, i}-\mathbf{v}_{i}\right),
$$

where $\mathbf{u}_{f, i}$ is the instantaneous fluid velocity interpolated at particle $i$, and $A_{s, i}$ is the projected area of the $i$-th spherical particle (or equivalent spherical particle for non-spherical particles). According to the Reynolds decomposition, the instantaneous fluid velocity is decomposed into the Reynolds-averaged component $\overline{\mathbf{u}}_{f, i}$ and the turbulent fluctuating component $\mathbf{u}_{f, i}^{\prime}$. In CFDEM-EIM, the Reynolds-averaged velocities are provided by the carrier fluid model. While the turbulent fluctuating component is modeled with an additional eddy-interaction closure (see Section 2.4). To generalize the drag coefficient for both spherical and non-spherical particles, the drag coefficient $C_{D}$ is given by (Haider and Levenspiel, 1989),

$$
C_{D}=f(\bar{\phi})\left[\frac{24}{R e_{p}}\left(1+A \cdot \operatorname{Re}_{p}{ }^{B}\right)+\frac{C}{1+D / R e_{p}}\right],
$$

where $\operatorname{Re}_{p}=(1-\bar{\phi})\left|\overline{\mathbf{u}}_{f, i}-\mathbf{v}_{i}\right| d_{i} / \nu_{f}$ is the particle Reynolds number, $\nu_{f}$ is the fluid kinematic viscosity, and $d_{i}$ is the diameter of the spherical particle or an equivalent sphere that has the same volume as the non-spherical particle. The four parameters $A, B, C$, and $D$ are proposed to be functions of particle sphericity, $\eta$, with

$$
\begin{aligned}
& A=\exp \left(2.3288-6.4581 \eta+2.4486 \eta^{2}\right), \\
& B=0.0964+0.5565 \eta, \\
& C=\exp \left(4.905-13.8944 \eta+18.4222 \eta^{2}-10.2599 \eta^{3}\right), \\
& D=\exp \left(1.4681+12.2584 \eta-20.7322 \eta^{2}+15.8855 \eta^{3}\right) .
\end{aligned}
$$

For spherical particles, $\eta=1$, and for nonspherical particles, $\eta<1$. In the drag coefficient (Eqn. 7), a correction for particle concentration, $f(\bar{\phi})$, is introduced to take into account 
the hindered settling effect (Di Felice, 1994),

$$
f(\bar{\phi})=(1-\bar{\phi})^{2-n}
$$

where, the empirical exponent, $n$, is related to the particle Reynolds number,

$$
n=3.7-0.65 \exp \left[-\frac{\left(1.5-\log _{10} R e_{p}\right)^{2}}{2}\right] .
$$

The local sediment concentration, $\bar{\phi}$, is calculated by averaging the sediment instantaneous sediment concentration within one CFD time step,

$$
\bar{\phi}=\frac{1}{N_{s}} \sum_{j=1}^{N_{s}} \phi_{j}
$$

where $N_{s}$ is number of DEM time steps within one CFD time step (see more details in Section 2.5), the divided volume fraction model (Goniva et al., 2012) is used for the instantaneous sediment concentration at each DEM time step, where the particle volumes are divided into 29 parts using the satellite points, and the volumes are distributed into the touched fluid grid cells. The model works well when particle size is similar to the mesh size.

\subsection{Fluid phase governing equations}

In contrast of the particle phase, the fluid phase is solved in an Eulerian framework and the coupled Euler-Lagrange system follows the so-called model "A" (e.g., Zhou et al. 2010). By further carrying out Reynolds-averaging, the fluid momentum equation may be written as,

$$
\frac{\partial \rho_{f}(1-\bar{\phi}) \overline{\mathbf{u}}_{f}}{\partial t}+\nabla \cdot\left[\rho_{f}(1-\bar{\phi}) \overline{\mathbf{u}}_{f} \overline{\mathbf{u}}_{f}\right]=(1-\bar{\phi}) \mathbf{f}_{x}-(1-\bar{\phi}) \nabla p+\nabla \cdot \tau_{f}+\rho_{f}(1-\bar{\phi}) \mathbf{g}+\overline{\mathbf{F}}_{d}
$$

where the overbar " - ' denotes the ensemble-averaged fields, $\rho_{f}$ is the fluid density. The first term on the right-hand-side (R.H.S.) is the external body force that drives the steady flow. The second term on R.H.S. is the pressure gradient force. $\tau_{f}$ is the total fluid stress tensor, which includes the viscous stress $\left(\tau_{\nu}\right)$ and the Reynolds stress $\left(\tau_{f t}\right)$. The last term 
on the R.H.S. is the sum of the drag force from the particles within the fluid grid volume $\left(V_{c e l l}\right)$, which must satisfy the Newton's 3rd law,

$$
\overline{\mathbf{F}}_{d}=-\frac{1}{N_{s} V_{\text {cell }}} \sum_{j=1}^{N_{s}} \sum_{i=1}^{N_{c e l l}} \mathbf{f}_{d, i} .
$$

The sediment concentration $(\bar{\phi})$ calculated directly by grid averaging in the DEM is usually not smooth, and averaging errors may depend on the averaging length scale (Simeonov et al., 2015). To ensure numerical stability, a diffusion model is often used to obtain a sufficiently smoothed concentration profile,

$$
\frac{\partial \bar{\phi}}{\partial t}=\nabla \cdot\left(D_{t} \nabla \bar{\phi}\right)
$$

The diffusion constant, $D_{t}$, is calculated as, $D_{t}=L_{d}^{2} / d t$, where $L_{d}$ is a length scale, and $d t$ is the fluid phase time step (i.e., CFD time step). The choice of length scale, $L_{d}=d$ is found to be stable and necessary when the fluid grid length is similar to or smaller than the particle diameter (Pirker et al., 2011; Capecelatro and Desjardins, 2013). Note that this smoothed concentration field is only used in the fluid governing equations and turbulence closures. The model results (mainly in Section 3 and 4 ) of the sediment concentration, sediment velocity and transport rate are directly obtained from the DEM part (i.e., not smoothed by the diffusion model). To ensure a stable calculation of conservation of mass, a mixture continuity equation for the incompressible fluid-particle system can be derived and is solved (Cheng et al., 2017a),

$$
\nabla \cdot\left[(1-\bar{\phi}) \overline{\mathbf{u}}_{f}+\bar{\phi} \overline{\mathbf{u}}_{s}\right]=0
$$

\subsection{Fluid turbulence modeling}

As briefly described in Eqn. 15, the total fluid stress tensor consists of the viscous stress $\left(\tau_{\nu}\right)$ and the Reynolds stress $\left(\tau_{f t}\right)$ :

$$
\tau_{f}=\tau_{\nu}+\tau_{f t}=\rho_{f}(1-\bar{\phi})\left[\left(\nu_{f}+\nu_{f t}\right)\left(\nabla \overline{\mathbf{u}}_{f}+\nabla^{T} \overline{\mathbf{u}}_{f}-\frac{2}{3} \mathbf{I} \nabla \cdot \overline{\mathbf{u}}_{f}\right)-\frac{2}{3} k_{f} \mathbf{I}\right],
$$

in which, the Reynolds stress in the Reynolds-averaged Eulerian fluid model may be written as,

$$
\tau_{f t}=\rho_{f}(1-\bar{\phi})\left[\nu_{f t}\left(\nabla \overline{\mathbf{u}}_{f}+\nabla^{T} \overline{\mathbf{u}}_{f}-\frac{2}{3} \mathbf{I} \nabla \cdot \overline{\mathbf{u}}_{f}\right)-\frac{2}{3} k_{f} \mathbf{I}\right]
$$


where $\mathbf{I}$ is a identity tensor, $\nabla^{T}$ is the transpose of gradient tensor, $\nu_{f t}$ is the turbulent eddy viscosity, and $k_{f}$ is the fluid turbulent kinetic energy (TKE). The eddy viscosity and TKE are modeled with a low Reynolds number version $k-\omega$ turbulence model (LRN $k-\omega$ closure (Wilcox, 1992)) modified for two-phase flows.

\subsubsection{Low Reynolds number corrected $k-\omega$ closure for two-phase flow}

In LRN $k-\omega$ closure, the low Reynolds number correction was introduced based on the local Reynolds number, $R e_{t}=k_{f} /\left(\nu_{f} \omega_{f}\right)$. With this correction, the LRN $k-\omega$ closure is capable of capturing transitional turbulent flow in the near-bed region. To take into account of the sediment effect on the flow turbulence, the sediment-turbulence interaction terms were added to both the transport equations for the fluid TKE $\left(k_{f}\right)$ and specific turbulent dissipation frequency $\left(\omega_{f}\right)$, similar to the approach suggested by Amoudry (2014) and Chauchat et al. (2017),

$$
\begin{gathered}
\frac{\partial k_{f}}{\partial t}+\overline{\mathbf{u}}_{f} \cdot \nabla k_{f}=\frac{\tau_{f t}}{\rho_{f}}: \nabla \overline{\mathbf{u}}_{f}+\nabla \cdot\left[\left(\nu_{f}+\frac{\nu_{f t}}{\sigma_{k}}\right) \nabla k_{f}\right]-C_{\mu}^{*} k_{f} \omega_{f} \\
-\frac{2 \beta(1-\lambda) \bar{\phi} k_{f}}{\rho_{f}(1-\bar{\phi})}-\frac{1}{(1-\bar{\phi})} \frac{\nu_{f t}}{\sigma_{c}}\left(\frac{\rho_{s}}{\rho_{f}}-1\right) \mathbf{g} \cdot \nabla \bar{\phi}
\end{gathered}
$$

where the operation ':' denotes the scalar product of two tensors. $C_{\mu}^{*}$ is model coefficients with low Reynolds number corrections based on the original coefficient $C_{\mu}$ (see table 1 ),

$$
C_{\mu}^{*}=C_{\mu} \frac{4 / 15+\left(R e_{t} / R e_{\beta}\right)^{4}}{1+\left(R e_{t} / R e_{\beta}\right)^{4}}
$$

where the model constant $R e_{\beta}=8$ is a critical Reynolds number.

Except for the last two terms on the R.H.S. of Eqn. (21), the rest of the terms in the present $k_{f}$ equation are essentially the same as those in the clear fluid TKE equation. The last term in Eqn. (21) represents the buoyancy term. For typical sediment concentration with an upward decaying profile, this term represents the well-known sediment-induced density stratification that can attenuate the fluid turbulence. The fourth term on the R.H.S. represents attenuation of TKE due to drag with $\beta$ calculated as,

$$
\beta=\frac{3}{4} \frac{\rho_{f} C_{D}\left|\mathbf{U}_{\mathbf{r}}\right|}{d}
$$


where $C_{D}$ is calculated by Eqn. (7) with particle Reynolds number, $R e_{p}=(1-\bar{\phi})\left|\mathbf{U}_{\mathbf{r}}\right| d / \nu_{f}$, in which $\left|\mathbf{U}_{\mathbf{r}}\right|$ is the magnitude of relative velocity seen by the fluid. Here, to better estimate $\mathbf{U}_{\mathbf{r}}$ in dilute condition, where instantaneous sediment concentration fluctuation is significant, a temporal average of the relative velocity is carried out,

$$
\mathbf{U}_{\mathbf{r}}=\frac{1}{t-t_{0}} \int_{t_{0}}^{t}\left(\overline{\mathbf{u}}_{f}-\overline{\mathbf{u}}_{s}\right) d t
$$

where $t_{0}$ is the starting time of the time average, and $t$ is the current run time of the simulation. For a steady sheet flow application, this time average procedure is representative of the ensemble-averaged relative velocity between fluid and sediment phases. Throughout the simulations in this study, the quasi-steady state is usually reached within $5 \mathrm{~s}$ of numerical simulations, thus we choose $t_{0}=5 \mathrm{~s}$. To quantify the effect of fluid-particle turbulence modulation, the parameter $\lambda$ is introduced by following Cheng et al. (2017a),

$$
\lambda=e^{-C_{s} \cdot S t},
$$

where $C_{s}$ is an empirical coefficient. $S t=t_{p} / t_{l}$, is the particle Stokes number, i.e., the ratio of the particle response time $\left(t_{p}=\rho_{s} / \beta\right)$ to the characteristic time scale of energetic eddies. In the literatures of Reynolds-averaged turbulence closures, the general expression for the eddy life time can be written as, $t_{l}=C_{t} /\left(C_{\mu} \omega_{f}\right)$, and the value of the coefficient $C_{t}$ ranges from 0.135 to 0.41 (Milojeviè, 1990), which is highly dependent on the flow conditions. From the preliminary numerical experiment, we found the the eddy life time is vital for the turbulence-sediment interaction, thus we chose the coefficient $C_{t}=1 / 6$ by following Cheng et al. (2017a), and the model coefficients associated with the eddy life time are left as model calibration. For example, the coefficient $C_{s}$ in Eqn. (25) is calibrated using the sheet flow experimental dataset (see Section 3.2) to match the flow hydrodynamics, and it was chosen to be $C_{s}=1$.

The balance equation for $\omega_{f}$ follows the original work of Wilcox (1992). However, for turbulence-particle sinteractions, similar damping terms as in the $k_{f}$ equation are included. The $\omega_{f}$ equation is written as,

$$
\begin{aligned}
\frac{\partial \omega_{f}}{\partial t} \quad+\overline{\mathbf{u}}_{f} \cdot \nabla \omega_{f}=C_{1 \omega}^{*} \frac{\omega_{f}}{k_{f}} \frac{\tau_{f t}}{\rho_{f}}: \nabla \overline{\mathbf{u}}_{f}+\nabla \cdot\left[\left(\nu_{f}+\frac{\nu_{f t}}{\sigma_{\omega}}\right) \nabla \omega_{f}\right]-C_{2 \omega} \omega_{f}{ }^{2} \\
-C_{3 \omega} \frac{2 \beta(1-\lambda) \bar{\phi} \omega_{f}}{\rho_{f}(1-\bar{\phi})}-C_{4 \omega} \frac{\omega_{f}}{k_{f}} \frac{1}{(1-\bar{\phi})} \frac{\nu_{f t}}{\sigma_{c}}\left(\frac{\rho_{s}}{\rho_{f}}-1\right) \mathbf{g} \cdot \nabla \bar{\phi}+\frac{\omega_{\text {bed }}}{T_{\text {relax }}} \Gamma(\bar{\phi}),
\end{aligned}
$$


where the fourth and fifth terms take into account of the sediment effect on the fluid turbulence through drag and buoyancy, respectively. The coefficients $C_{1 \omega}^{*}$ is also modulated using the local turbulent Reynolds number as,

$$
C_{1 \omega}^{*}=C_{1 \omega} \frac{1}{\alpha^{*}} \frac{\alpha_{0}+R e_{t} / R e_{\omega}}{1+R e_{t} / R e_{\omega}}
$$

where $\alpha^{*}$ is a damping function based on $R e_{t}$,

$$
\alpha^{*}=\frac{\alpha_{0}^{*}+R e_{t} / R e_{k}}{1+R e_{t} / R e_{k}} .
$$

where $\alpha_{0}^{*}$ and $R e_{k}$ are model coefficient for the low Reynolds number corrections. The model constant $C_{1 \omega}, C_{2 \omega}, \sigma_{k}, \sigma_{\omega}, R e_{k}, R e_{\omega}$ and $\alpha_{0}$ are similar to the closure coefficients suggested by Guizien et al. (2003) (see Table 1). The coefficient of the buoyancy term, $C_{4 \omega}=0$ is chosen for stable stratification applicable for steady sheet flow (Rodi, 1987). Through a series of sensitivity test, we found that the modeled flow velocities are also sensitive to the coefficient $C_{3 \omega}$, and the optimum value of $C_{3 \omega}$ is 0.14 , which is close to the value 0.2 suggested by Amoudry (2014). A full list of the coefficients associated with the low Reynolds number $k-\omega$ model used in this study is presented in Table 1 .

\begin{tabular}{ccccccccccccc}
\hline$\alpha_{0}$ & $\alpha_{0}^{*}$ & $R e_{k}$ & $R e_{\omega}$ & $R e_{\beta}$ & $C_{\mu}$ & $\sigma_{k}$ & $\sigma_{\omega}$ & $C_{s}$ & $C_{1 \omega}$ & $C_{2 \omega}$ & $C_{3 \omega}$ & $C_{4 \omega}$ \\
\hline $1 / 9$ & 0.024 & 6 & 2.95 & 8 & 0.09 & 2.0 & 2.0 & 1.0 & 0.52 & 0.072 & 0.14 & 0 \\
\hline
\end{tabular}

Table 1: List of coefficients in the LRN $k-\omega$ equations for two-phase flows.

Finally, the turbulent eddy viscosity $\nu_{f t}$ is calculated by the fluid turbulence kinetic energy $k_{f}$ (TKE) and specific turbulence dissipation rate $\omega_{f}$,

$$
\nu_{f t}=\alpha^{*} \frac{k_{f}}{\omega_{f}} .
$$

It shall be noted that the LRN $k-\omega$ can be reduced to the original $k-\omega$ model (Wilcox, 1993) in the fully turbulent region when the local Reynolds number is sufficiently high compared with the critical Reynolds numbers. 


\subsubsection{Smooth and Rough wall functions}

The wall functions for a smooth bed and rough bed are both relevant to the present study. For clear fluid or dilute suspension, such as the experiment of Muste et al. (2005) to be discussed in Section 3.1, a smooth wall is exposed and the $\omega_{f}$ value in the viscous sublayer scales with $1 / z^{2}$, where $z$ is the distance to the bottom wall boundary. As a result, $\omega_{f}$ goes to infinity at the wall boundary. In the numerical implementation, a finite value of $\omega_{f}$ is imposed to the first grid above the solid smooth wall, and the following bottom boundary condition is specified (Menter and Esch, 2001; Bredberg et al., 2000),

$$
\omega_{\text {wall }}=\sqrt{\omega_{v i s}^{2}+\omega_{\text {log }}^{2}},
$$

with the $\omega_{\text {wall }}$ value specified as a blend function of the values in the viscous sublayer $\left(\omega_{\text {vis }}\right)$ and logarithmic layer $\left(\omega_{\text {log }}\right)$,

$$
\omega_{v i s}=\frac{6 \nu_{f}}{0.075 z_{o}^{2}}, \omega_{l o g}=\frac{u_{*}}{\sqrt{C_{\mu}} \kappa z_{o}},
$$

where $\kappa=0.41$ is the von Karman constant, and the bottom frictional velocity is calculated as $u_{*}=\sqrt{\left(\nu_{f}+\nu_{f t}\right)\left|\partial \bar{u}_{f} / \partial z\right|}$ at the wall boundary. It was found that this formulation of bottom boundary condition for smooth wall is robust for low to high Reynolds number turbulent boundary layer flows.

On the other hand, the bed is covered with a thick layer of sediment particles in sheet flow condition, and the particles imposes a rough wall boundary to the flow above the bed. However, the location of the bed in sediment transport is difficult to determine as a priori due to possible erosion processes. To avoid this complexity, the last term on the R.H.S. of Eqn. (26) is proposed to impose a desired value of specific turbulence dissipation rate, $\omega_{b e d}$, in the sediment bed, and $\Gamma(\bar{\phi})$ is a step-like function of sediment concentration,

$$
\Gamma(\bar{\phi})=\frac{\tanh \left[500\left(\bar{\phi}-\phi_{b}\right)\right]+1}{2},
$$

where $\phi_{b}$ should be specified as the sediment concentration in the bed, so that the $\omega_{f}$ value is only imposed inside the sediment bed. In this study, we choose $\phi_{b}=0.55$. An intrinsic relaxation timescale is used for $T_{\text {relax }}$, which sums the proper timescale on the 
R.H.S. of the $\omega_{f}$ equation,

$$
\frac{1}{T_{\text {relax }}}=C_{2 \omega} \omega_{f}+C_{3 \omega} \frac{2 \beta(1-\lambda) \bar{\phi}}{\rho_{f}(1-\bar{\phi})}+C_{4 \omega} \frac{1}{k_{f}} \frac{1}{(1-\bar{\phi})} \frac{\nu_{f t}}{\sigma_{c}}\left(\frac{\rho_{s}}{\rho_{f}}-1\right) \mathbf{g} \cdot \nabla \bar{\phi} .
$$

It shall be noted that the relaxation time scale proposed here is positive in sheet flow applications. For specific energy dissipation frequency $\omega_{\text {bed }}$ inside the bed, the rough wall value can be specified as (Wilcox, 1988),

$$
\omega_{\text {bed }}=S_{r} \frac{u_{*}^{2}}{\nu_{f}}
$$

where $u_{*}$ is the bottom frictional velocity at the bed interface specified based on the flow forcing to drive the steady channel flow and $S_{r}$ is a parameter depending on the bed roughness,

$$
S_{r}=\left\{\begin{array}{r}
\left(\frac{200}{k_{s}^{+}}\right)^{2}, k_{s}^{+}<5 \\
\frac{K_{r}}{k_{s}^{+}}+\left[\left(\frac{200}{k_{s}^{+}}\right)^{2}-\frac{K_{r}}{k_{s}^{+}}\right] e^{\left(5-k_{s}^{+}\right)}, k_{s}^{+} \geq 5
\end{array},\right.
$$

where $k_{s}^{+}=k_{s} u_{*} / \nu_{f}$ is the normalized wall roughness in wall units, and $k_{s}$ is the Nikuradse's equivalent sand roughness, which is related with the sand grain size, $k_{s}=2.5 d$. The original coefficient $K_{r}$ is 100 as suggested by Wilcox (1988), however, Fuhrman et al. (2010) proposed that this coefficient needs to be reduced to $K_{r}=80$ to match the law of wall. Therefore, $K_{r}=80$ is used throughout this paper.

\subsection{Eddy interaction model}

The drag force (Eqn. 6) in the particle momentum equation depends on the instantaneous fluid velocity. However, only the Reynolds-averaged fluid velocity $\left(\overline{\mathbf{u}}_{f, i}\right)$ is solved and hence an additional closure model for the fluid velocity fluctuation in turbulent flow $\left(\mathbf{u}_{f, i}^{\prime}\right)$ is required. Appropriate consideration of particle dispersion by turbulent eddies provides a key suspension mechanism in sediment transport (i.e., turbulent suspension). Following Graham and James (1996), particle dispersion by turbulence can be modeled with a stochastic Eddy Interaction Model (EIM), and a series of random Lagrangian velocities can be used to represent the fluid turbulent motions, i.e. $u_{f, i}^{\prime}=U_{i}^{t} \sigma_{1}, v_{f, i}^{\prime}=V_{i}^{t} \sigma_{2}$, and $w_{f, i}^{\prime}=W_{i}^{t} \sigma_{3}$, where $\sigma_{1,2,3}$ are Gaussian random numbers with a zero mean value and a standard deviation of unity. In this study, the velocity fluctuations are calculated using 
the fluid turbulent kinetic energy, $U_{i}^{t}=V_{i}^{t}=W_{i}^{t}=\sqrt{2 k_{f, i} / 3}$, where $k_{f, i}$ is interpolated turbulence kinetic energy at the mass center of particle $i$. It is possible to model the anisotropic velocity fluctuations in three directions, however, to be consistent with the two-equation turbulence-averaged models (LRN $k-\omega$ closure), the turbulent fluctuations are assumed to be isotropic.

In the eddy interaction model, the velocity fluctuations (i.e., $\left.U_{i}^{t}, V_{i}^{t}, W_{i}^{t}\right)$ are updated every step with the particle position. However, the random numbers $\sigma_{1,2,3}$ remained unchanged until the eddy interaction time $t_{I}$ is exceeded, which is determined either when a particle has completely crossed a turbulent eddy or remains in an eddy but exceeds the eddy life time. The mean life time of the turbulent eddy can be estimated as $T_{l, i}=\left(6 C_{\mu} \omega_{f, i}\right)^{-1}$ in the LRN $k-\omega$ model. However, the instantaneous turbulent eddy life time is of random-like nature (Kallio and Reeks, 1989; Mehrotra et al., 1998) and it is estimated as,

$$
t_{e, i}=-C_{0} \ln (1-\xi) T_{l, i}
$$

where $\xi$ is the random number ranging from 0 to 1 . As discussed in Section 2.3, due to the uncertainties in the parameterization of the eddy life time, the coefficient $C_{0}$ is introduced as a constant for model calibration (see Section 3.1). As a result, the turbulent eddy length $l_{e}$ can be estimated as $l_{e, i}=t_{e, i} \sqrt{2 k_{f, i} / 3}$. With the estimation of the turbulent eddy length $l_{e}$, the eddy crossing time for a particle can be computed as (Gosman and Loannides, 1983),

$$
t_{c, i}=-t_{p, i} \ln \left(1-\frac{l_{e, i}}{\left|\mathbf{v}_{i}-\overline{\mathbf{u}}_{f, i}\right| t_{p, i}}\right),
$$

where $t_{p, i}$ is the particle response time calculated as $t_{p, i}=4 \rho_{s, i} d_{i} /\left(3 \rho_{f}\left|\mathbf{v}_{i}-\overline{\mathbf{u}}_{f, i}\right| C_{D}\right)$. It is noted that Eqn. (37) is only evaluated when $l_{e, i}<\left|\mathbf{v}_{i}-\overline{\mathbf{u}}_{f, i}\right| t_{p, i}$, and the eddy interaction time $t_{I, i}$ is the minimum between eddy lifetime $t_{e, i}$ and eddy crossing time $t_{c, i}$. Once the time interval exceeds $t_{I, i}$, the particle $i$ enters another turbulent eddy, i.e., the gaussian random numbers $\sigma_{1,2,3}$ are re-evaluated every interval $t_{I, i}=\min \left\{t_{e, i}, t_{c, i}\right\}$. 


\subsection{CFD-DEM coupling procedure}

In the present Euler-Lagrange modeling framework, the coupling between the fluid phase and sediment phase utilizes the open source code CFDEM (Goniva et al., 2012), which couples the Finite-volume CFD toolbox OpenFOAM (Weller, 2002) with the DEM solver LIGGGHTS (Kloss et al., 2012). At the beginning of the simulation, the particle positions and velocities are initialized in the DEM module, and the fluid velocity and turbulence quantities are initialized in the CFD module. The loop of the CFD-DEM coupling begins with the update of particle positions and velocities for $N_{s}$ DEM time steps within one fluid time step $(d t)$, in which the time step $d t_{s}$ in the DEM module is related to the fluid time step by $d t_{s}=d t / N_{s}$. In the contact force model, the energy stored in the collision increases rapidly with the overlapping length of particles, thus the time step $d t_{s}$ should be sufficiently small to avoid the unphysical energy generation due to particle contacts. In this study, the following three criteria are used to determine $d t_{s}$ :

(1) The overlap length $\delta_{n}$ is smaller than $0.5 \%$ of particle diameter $d$, i.e., $d t_{s}<$ $0.005 d / V_{r n}$, where $V_{r n}$ is normal component of the relative velocity to the contact face between two contacting particles.

(2) To capture the energy transmission in the solid particles, the time step $d t_{s}$ is chosen to be small enough compared with the Rayleigh timescale $T_{r}$, where $T_{r}=$ $\pi r \sqrt{\rho_{s} / G}(0.163 \nu+0.8766)^{-1}$ and $G$ is the shear modulus. $G$ is further related to the Young's modulus $E$ and the Poisson ratio $v$ as $2 G(1+v)=E$.

(3) $d t_{s}$ is required to be smaller than the Hertzian contact time in order to capture the contact process. The Hertzian contact time is the duration of a pair of particles in contact, which can be estimated as, $T_{c}=2.87\left(m^{* 2} / r^{*} E^{* 2} V_{r n}\right)^{1 / 5}$, where $r^{*}=$ $\left(\frac{1}{r_{i}}+\frac{1}{r_{j}}\right)^{-1}, m^{*}=\left(\frac{1}{m_{i}}+\frac{1}{m_{j}}\right)^{-1}$, and $E^{*}=\left(\frac{1-\nu_{i}^{2}}{E_{i}}+\frac{1-\nu_{j}^{2}}{E_{j}}\right)^{-1}$. For a contact between a sphere particle $i$ with wall $j$, the same relationship applies to $E^{*}$, whereas $r^{*}=r_{i}$ and $m^{*}=m_{i}$.

The $d t_{s}$ is constant throughout the simulation once appropriately chosen to satisfy the above criteria, and the particle velocities are updated every $d t_{s}$, where the forces acting on 
each particles are solved according to Eqn. (4). In the calculation of drag forces, the eddy interaction model is implemented to model the turbulence-induced sediment suspensions, where a fluctuating component of velocities are introduced to the drag forces through a stochastic procedure, which is outlined as follows:

(a) Initially at $t=0$, the time marker $t_{m a r k, i}$, and eddy interaction time $t_{I, i}$ are set to zero for each particle.

(b) Random numbers $\sigma_{1,2,3}$ are generated and the fluid velocity fluctuation $u_{f, i}^{\prime}, v_{f, i}^{\prime}, w_{f, i}^{\prime}$ are updated. The drag forces are then calculated using Eqn. (6).

(c) The following two scenarios are considered:

(i) If $\left(t-t_{\text {mark }, i}\right) \geq t_{I, i}$, the particle enters a new turbulent eddy, and then new Gaussian random number $\sigma_{1,2,3}$ are generated, and fluid fluctuations are updated with the new values of $\sigma_{1,2,3}$. Both $t_{m a r k, i}$ and $t_{I, i}$ are updated to the current values.

(ii) Else if $\left(t-t_{\text {mark }, i}\right)<t_{I, i}$, the particle remains in the same eddy, thus the existing Gaussian random numbers are retained, and $t_{m a r k, i}$ and $t_{I, i}$ remains unchanged. However, the fluid fluctuations are updated with new particle positions (i.e., new $\left.k_{f, i}\right)$.

After solving the particle velocities and positions, the particle informations are communicated to the fluid phase. However, prior to solving the fluid equations, the diffusion model of Sun and Xiao (2016a) (see Eqn. 17) is applied to the sediment concentration to obtain a smooth profile. The fluid phase is computed in a similar way as the Eulerian two-phase flow model SedFOAM (Cheng et al. 2017a). The fluid momentum equation in Eqn. 15 is solved over a collocated grid arrangement, in which the velocities and pressure are stored in the cell centers. The convection terms (including the $k-\omega$ equations) are discretized using a total variation diminishing (TVD) scheme based on a Sweby limiter (Sweby, 1984). The second-order central scheme is used for the diffusion terms. 
For the temporal integration, a first-order implicit Euler scheme is used. The PISO (Pressure Implicit Splitting Operation) algorithm is used for the velocity-pressure decoupling, so that the continuity equation $(18)$ is satisfied. More details on the numerical solution procedures for the fluid solver can be found in Rusche (2002).

\section{Model Results}

Through preliminary numerical experiments, we confirmed that the modeled sediment concentration profile is sensitive to the prediction of fluid TKE and the coefficient $C_{0}$ in estimating the turbulent eddy life time in the eddy interaction model (see Eqn. 36). This is somewhat expected as the turbulent intensity and the eddy interaction time are the main factors differentiating the present stochastic procedure for modeling turbulent diffusion from incoherent random motions. Therefore, we first validated the turbulence closure with direct numerical simulation (DNS) of clear fluid turbulent channel flow. After establishing the accuracy of the turbulence closure for clear fluid, the coefficient $C_{0}$ in the eddy interaction model is calibrated with the dilute suspension experiment of Kiger and Pan (2002), where the velocity, sediment concentration and Reynolds shear stress profiles are measured for sand in a steady channel flow over a smooth bed (starved bed). The calibrated model is then applied to predict the suspended sand concentration and turbulence of another similar dilute suspension experiment reported by Muste et al. (2005). Because the sediment concentration is very dilute $(<1 \%)$ and there is negligible deposit on the bed, these datasets allow us to solely calibrate the $C_{0}$ value in the eddy interaction model without complication from intergranular interactions. After the calibration, the „model is applied to the steady sheet flow experimental configuration of Revil-Baudard et al. (2015). A sensitivity analysis of the model results to the $C_{0}$ value is investigated in detail to illustrate the effects on the turbulent suspension in steady sheet flow. The capability of the present CFDEM-EIM is further demonstrated by comparing predictions of sediment transport rate and transport layer thickness with classical empirical formula. 


\subsection{Model calibration of dilute suspension in steady channel flow}

Firstly, the LRN $k-\omega$ turbulence closure is validated against the DNS dataset of Moser et al. (1999) for a clear fluid steady wall-bounded channel flow at a Reynolds number of $R e_{\tau}=u_{*} h / \nu_{f}=570$ (Moser et al., 1999), where $h$ is the channel half-width. We carried out a 1DV numerical simulation at the same Reynolds number with a vertical domain height $h=0.02 \mathrm{~m}$. A shear-free symmetric boundary condition is used at the top boundary, while the bottom boundary condition is a no-slip wall. The standard smooth wall functions for $k$ and $\omega$ are used at the bottom wall boundary (see Eqn. 31). In both $x$ and $y$ directions, periodic boundaries are used and only one grid cell is used in these two directions with a grid size (domain size) of $L_{x}=L_{y}=0.02 \mathrm{~m}$. The vertical domain is discretized into 168 grid cells with a uniform grid size $\Delta_{z}=0.122 \mathrm{~mm}$. The flow is driven by a mean pressure gradient of $f_{x}=43.5 \mathrm{~Pa} / \mathrm{m}$, so that the bottom frictional velocity is $u_{*}=0.0285 \mathrm{~m} / \mathrm{s}$. The distance of the first grid center to the bottom boundary patch corresponds to a wall unit $\Delta_{z}^{+}=0.5 u_{*} \Delta_{z} / \nu_{f}=1.76$. Therefore, the first cell center is within the viscous sublayer.

The comparisons of the mean Reynolds shear stress, velocity and TKE profiles between the LRN $k-\omega$ model and DNS data are shown in Fig. 1. Very good agreements on all three profiles are obtained, especially the velocity profile and Reynolds shear stress. The agreement in the Reynolds shear stress profile confirms that the flow has reached a quasi-steady state and the flow condition is similar to the DNS simulation of Moser et al. (1999). Meanwhile, it is evident that the overall shear stress follows a linear profile $\tau_{\text {tot }}=u_{*}^{2}(1-z / h)$ in the range of $z / h>0.1$ (dashed curve in Fig. 1 b). The modeled TKE is also remarkably close to the DNS data. It is evident that the LRN $k-\omega$ model is able to resolve the peak of turbulent kinetic energy near the bottom wall (around $z=0.02 h$ ), even though the peak value from the LRN $k-\omega$ closure $\left(4.4 u_{*}^{2}\right)$ is slightly smaller than the DNS data $\left(4.75 u_{*}^{2}\right)$.

Kiger and Pan (2002) later conducted a sediment-laden turbulent flow experiment at a similar Reynolds number as Moser et al. (1999). The data of Kiger and Pan (2002) can be further used to calibrate the $C_{0}$ coefficient in the EIM. In the experiment, the half channel 

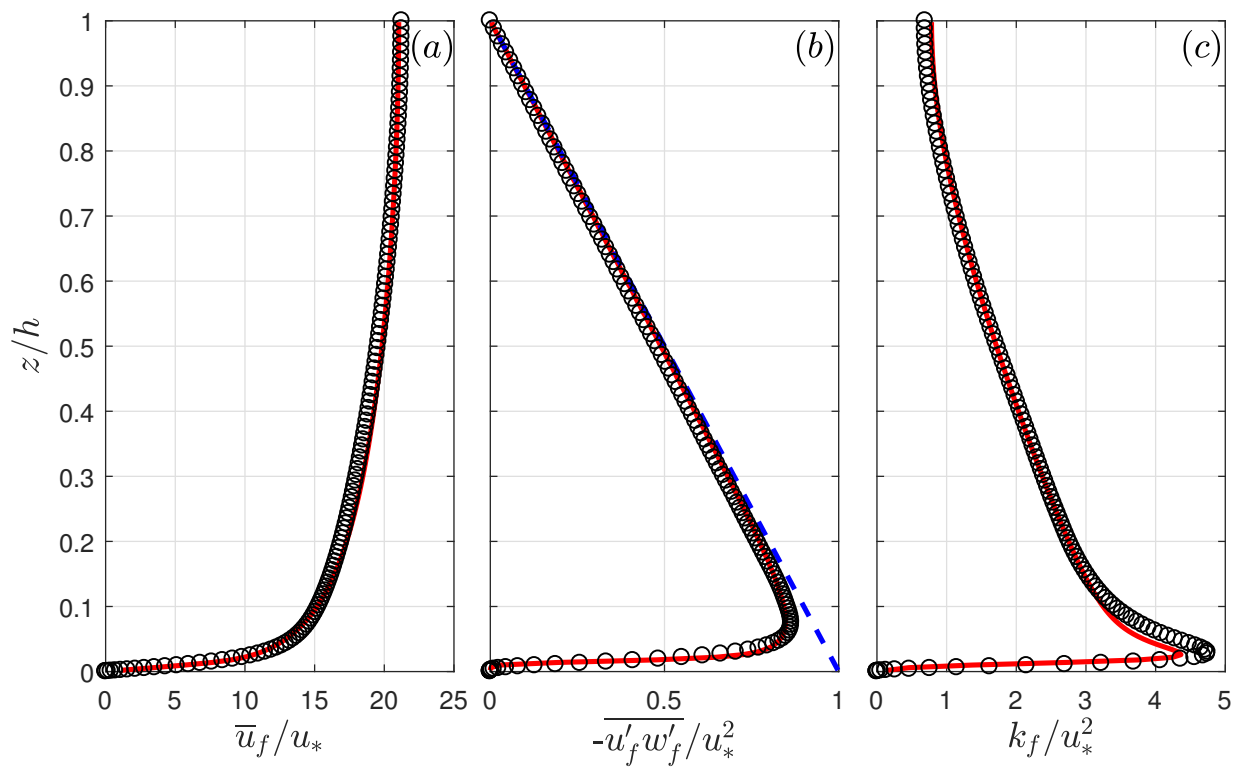

Figure 1: The comparison of non-dimensionalized (a) streamwise velocity profile $\left(\bar{u}_{f} / u_{*}\right)$, (b) Reynolds shear stress profile $\left(-\overline{u_{f}^{\prime} w_{f}^{\prime}} / u_{*}^{2}\right)$, and (c) TKE profile $\left(k_{f} / u_{*}^{2}\right)$ between LRN $k-\omega$ closure (solid curves) and DNS data (symbols) of Moser et al. (1999). In panel (b), the dashed curve denotes a linear fit of the total shear stress, $\tau_{\text {tot }}=u_{*}^{2}(1-z / h)$.

height is $h=0.02 \mathrm{~m}$, which is the same as the clear fluid simulation at $R e_{\tau}=570$, and hence we kept the same domain setup and boundary conditions as the clear fluid 1DV simulation. In the DEM implementation, the particles are tracked in a meshless 3D domain (domain size is the same as in the CFD). The lateral boundaries in DEM are periodic, while the wall boundary was used for both the top and bottom boundaries to conserve the number of particles in the domain. The sediments are spherical particles with a density of $\rho_{s}=2605 \mathrm{~kg} / \mathrm{m}^{3}$, and the grain diameter is $d=0.195 \mathrm{~mm}$. The particle settling velocity is about $0.024 \mathrm{~m} / \mathrm{s}$, which corresponds to a shape factor $\eta=1$ in the drag model (see Eqn. 8 11). The domain averaged sediment volumetric concentration in the experiment is $\Phi=2.3 \times 10^{-4}$. To match the domain-averaged sediment concentration in Kiger and Pan (2002), a total of $N=476$ particles are simulated in the DEM.

To calibrate the $C_{0}$ value in the EIM, we carried out four simulations with different $C_{0}$ values, $C_{0}=1,2,3,4$. The resulting profiles of the streamwise velocity, TKE and sediment concentration are compared with the measured data of Kiger and Pan (2002) 

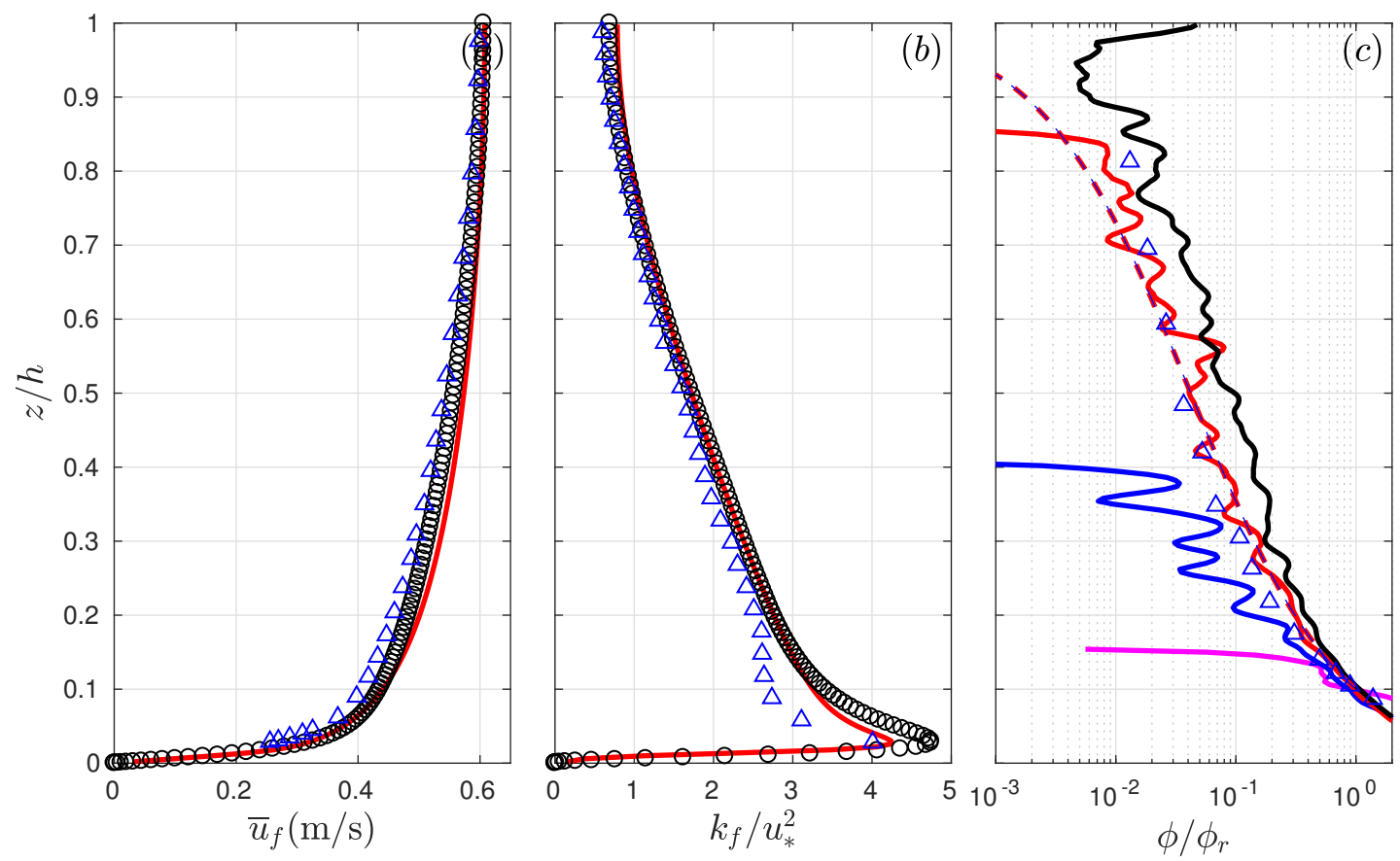

Figure 2: The comparison of (a) fluid velocity profile, (b) nondimensional fluid turbulent kinetic energy $\left(k_{f}\right.$, normalized by $\left.u_{*}^{2}\right)$ and (c) normalized concentration profile between model results (solid curves) and measured (or DNS) data (symbols). In all panels, the triangle symbols are measured data from Kiger and Pan (2002), and the DNS data of Moser et al. (1999) is shown as circle symbols in panel (a) and (b). In panel (c), sediment concentrations are plotted in semi-logrithmic scale. The solid curves corresponds to $C_{0}=1$ (magenta), $C_{0}=2$ (blue), $C_{0}=3$ (red) and $C_{0}=4$ (black). The dashed curve is the fitted Rouse profile with $R o=1.44$.

and clear fluid DNS data of Moser et al. (1999) in Fig. 2, Due to the dilute sediment concentration in the domain, the numerical results of the streamwise velocity profile are not very sensitive to the $C_{0}$ values, so only the velocity profiles corresponding to $C_{0}=3$ is shown. We notice that the measured velocity profile differs slightly from the DNS data, possibly due to the effect of the presence of sediment in the water column and/or measurement uncertainties. In addition, the averaged particle velocity profile (not shown) is very close to the fluid velocity. The measured data of the turbulent intensity is only available for the streamwise $\left(u_{r m s}^{\prime}\right)$ and vertical $\left(w_{r m s}^{\prime}\right)$ velocity fluctuations. In order to compare the turbulence kinetic energy of numerical results and measured data, the 
„spanwise velocity fluctuation is reconstructed following the relationship suggested by Jha and Bombardelli (2009), $v_{r m s}^{\prime}=0.3 u_{*}-0.6 u_{r m s}^{\prime}$. Thus the turbulent kinetic energy in the experiment can be estimated by $k_{f}=\left(u_{r m s}^{\prime 2}+v_{r m s}^{\prime 2}+w_{r m s}^{\prime 2}\right) / 2$. The model results also predict slightly smaller turbulence kinetic energy compared with clear fluid counterpart, but the reduction is very small due to dilute sediment concentration. Overall, the velocity and turbulence kinetic energy profiles are in good agreement with the measured data.

The sediment concentration profiles corresponding to different $C_{0}$ values are presented in Fig. 2p (solid curves) and they can be compared with measured data (symbols in Fig. 2c). It is evident that the suspended sediment concentration is strongly affected by the coefficient $C_{0}$. In general, more significant sediment suspension is obtained with a larger $C_{0}$ value. Clearly, a $C_{0}$ value of 1 under-predicts the suspended sediment concentration, and almost all the sediment particles accumulate near the bottom $(z / h<0.15$, see magenta curve in Fig. 22). When the $C_{0}$ value is increased to $C_{0}=2$, considerably more sediments are suspended, however, the resulting sediment concentration remains to be lower than the measured data. The optimum $C_{0}$ value is found to be $C_{0}=3$, and the resulting sediment concentration profile is in good agreement with the measured data. Finally, using $C_{0}=4$ clearly over-predicts sediment concentration. It is well-known that the sediment concentration profile in a steady turbulent channel flow follows the Rouse profile (Vanoni, 2006),

$$
\frac{\bar{\phi}}{\phi_{r}}=\left(\frac{h-z}{z} \frac{z_{r}}{h-z_{r}}\right)^{-R o},
$$

where $R o=w_{s} S c /\left(\kappa u_{*}\right)$ is the Rouse number, in which the Schmidt number $S c$ is the ratio of turbulent eddy viscosity over the sediment diffusivity. $z_{r}$ is the reference location above the bed, and $\phi_{r}$ is the concentration at the reference location. We choose the reference location to be $z_{r}=0.1 \mathrm{~h}$, corresponding to the lowest elevation that the Reynolds shear stress follows a linear profile. The dashed curve in Fig. $2 \mathrm{k}$ shows the fitted Rouse profile to the measured data with the Rouse number $R o=1.44$. It is evident that both the measured data and the numerical result with $C_{0}=3$ match the Rouse profile very well.

The calibrated $C_{0}$ is further applied to another similar dilute suspension experiment reported by Muste et al. (2005, see Table 2). The flow is driven by a prescribed pressure 
gradient force in order to match the bottom friction velocity of $u_{*}=0.042 \mathrm{~m} / \mathrm{s}$ in a flow depth of $h=0.021 \mathrm{~m}$. The sand density is $\rho_{s}=2650 \mathrm{~kg} / \mathrm{m}^{3}$ and the grain diameter is $d=0.23 \mathrm{~mm}$. The measured settling velocity is about $2.4 \mathrm{~cm} / \mathrm{s}$, which correspond to a shape factor of $\eta=0.644$ (see Eqn. 8,11). A similar numerical setup as the simulation of Kiger and Pan (2002) is used, except that the domain height is $h=0.021 \mathrm{~m}$ to match the experimental condition. The streamwise and spanwise domain lengths are specified to be $L_{x}=L_{y}=100 d$. In the vertical direction, uniform grid sizes are used with $N_{z}=210$ grids to resolve the entire flow depth, and the first grid center above the bottom wall corresponds to a wall unit $\Delta_{z}^{+}=1.05$. A total number of particles used in the DEM is $N=803$, which matches the domain averaged concentration $\Phi=4.6 \times 10^{-4}$.

\begin{tabular}{|c|c|c|c|c|c|c|c|}
\hline \multicolumn{2}{|r|}{ Cases } & $d(\mathrm{~mm})$ & $\rho_{s}\left(\mathrm{~kg} / \mathrm{m}^{3}\right)$ & $w_{s}(\mathrm{~cm} / \mathrm{s})$ & $u_{*}(\mathrm{~cm} / \mathrm{s})$ & $\Phi \times 10^{3}$ & $N$ \\
\hline Kiger & and Pan (2002) & 0.195 & 2605 & 2.4 & 2.85 & 0.23 & 476 \\
\hline NS1 in I & Muste et al. (2005) & 0.23 & 2650 & 2.4 & 4.2 & 0.46 & 803 \\
\hline
\end{tabular}

Table 2: List of numerical simulations of dilute sand suspension in steady channel flows.

The model results of velocity profile, concentration profile and TKE $\left(k_{f}\right)$ profile with $C_{0}=3$ are compared with the measured data in Fig. 3. The resulting fluid velocity profile (Fig. 3a) matches the measured data reasonably well, except that the velocity magnitude is slightly over-predicted in the range of $0.1<z / h<0.5$. The normalized sediment concentration (normalized by the mean concentration $\phi_{r}$ at the reference location $\left.z_{r} / h=0.1\right)$ shows that the suspended sediment concentration profile is similar to the measured data as well as the Rouse profile with a Rouse number $R o=0.86$ (dashed curve in Fig. 3b). In Fig. 3k, the numerical result of TKE is compared with the measured data. The measured data of the turbulent intensity is reconstructed in the same way as the measurement of Kiger and Pan (2002). Overall, the magnitude of the turbulent kinetic energy is smaller than the measured data by no more than $30 \%$. However, the vertical profile shape is reproduced well by the model.

In summary, the LRN $k-\omega$ model is validated using a clear fluid DNS dataset of Moser 

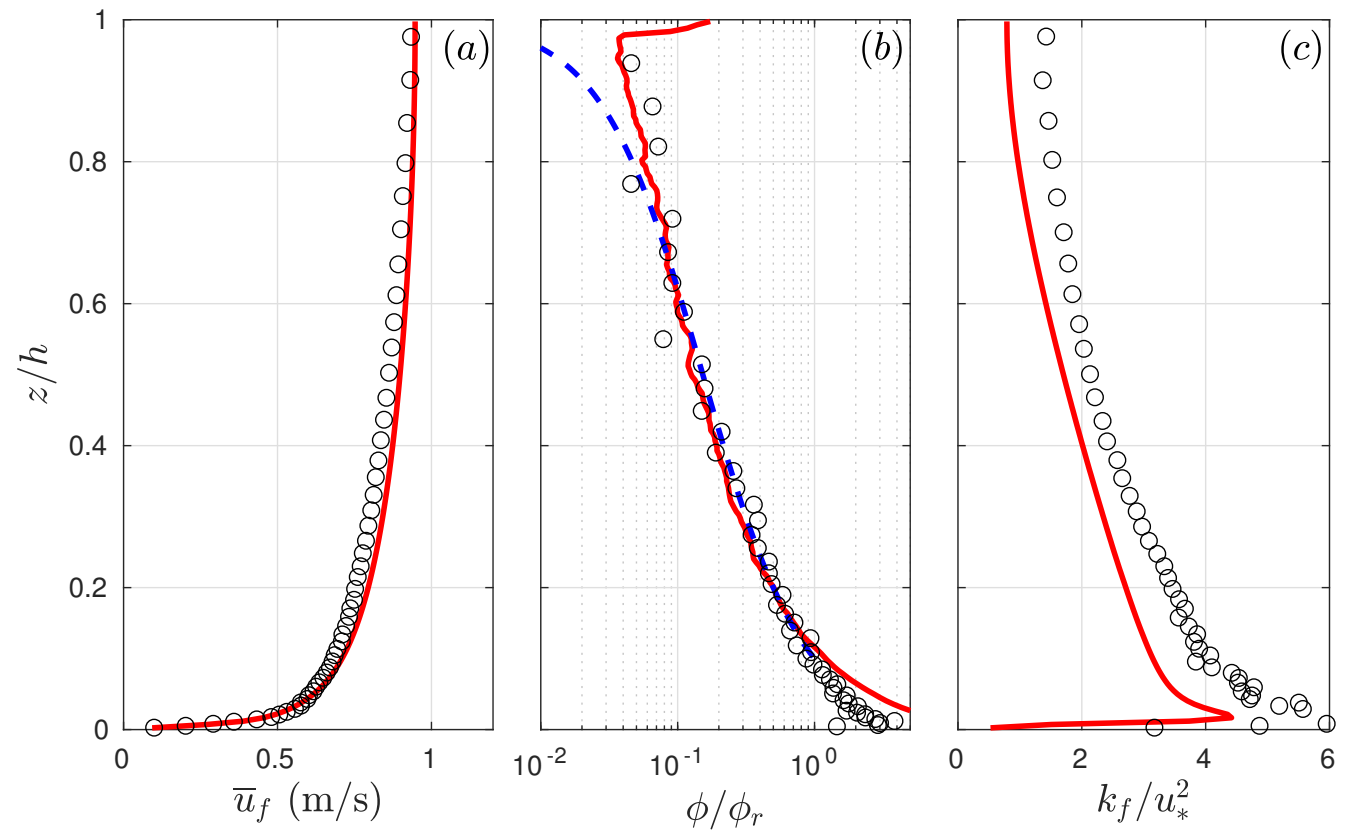

Figure 3: The comparison of (a) fluid velocity profile, (b) normalized concentration profile, and (c) fluid turbulent kinetic energy $\left(k_{f}\right.$, normalized by $\left.u_{*}^{2}\right)$ between model results and measured data of case NS1 in Muste et al. (2005). In all panels, the symbols represent the measure data in Muste et al. (2005), and the solid curves are model results. In panel (b), sediment concentrations are plotted in semi-log scale. The dashed curve is the Rouse profile with $R o=0.86$.

et al. (1999) and the eddy interaction model is calibrated by using the measurements from Kiger and Pan (2002) and Muste et al. (2005). It is found that the optimum $C_{0}$ value that matches the measured concentration profiles for both experiments is $C_{0}=3.0$, while $C_{0}<3.0$ underestimated the suspended sediment concentration. Therefore, this calibrated $C_{0}$ value is applied to the sheet flow applications in the following subsection.

\subsection{Steady sheet flow}

In this section, we further apply CFDEM-EIM to model steady sheet flow, where both bedload (inter-granular interaction dominant) and suspended load (turbulent suspension dominant) are important. The laboratory experiments reported by Revil-Baudard et al. (2015, 2016), which include a steady flow over a rough fixed bed ("FB") and a steady sheet flow (mobile bed, "MB") were used for model validation. The flow condition and 
sediment properties are summarized in Table 3. The sediment particles are irregularly shaped with density $\rho_{s}=1192 \mathrm{~kg} / \mathrm{m}^{3}$, and median grain diameter $d=3 \mathrm{~mm}$. The resulting mean settling velocity is measured to be $w_{s}=5.59 \mathrm{~cm} / \mathrm{s}$. Similar to the case NS1 in Muste et al. (2005), we used a sphericity of $\eta=0.594$ to match the settling velocity with the experiment, while the original grain size $d$ is retained in the DEM contact model. In the DEM model, the Young's modulus of particles is specified as $E=5 \times 10^{6} \mathrm{~Pa}$, the restitution coefficient is $e=0.5$, the Coulomb friction coefficient is $\mu_{c}=0.5$ and the poison ratio is $\nu=0.45$. In the fixed bed ("FB") experiment, these particles are glued to the bed forming a single layer rough elements, while the bed is covered by thick layers of particles in the "MB" case, and the particles are free to move.

\begin{tabular}{cccccccc}
\hline Cases & $h(\mathrm{~m})$ & $u_{*}(\mathrm{~cm} / \mathrm{s})$ & $\rho_{f}\left(\mathrm{~kg} / \mathrm{m}^{3}\right)$ & $\nu_{f}\left(\mathrm{~m}^{2} / \mathrm{s}\right)$ & $d(\mathrm{~mm})$ & $s=\rho_{s} / \rho_{f}$ & $w_{s}(\mathrm{~cm} / \mathrm{s})$ \\
\hline $\mathrm{FB}$ & 0.105 & 5.2 & 1000 & $10^{-6}$ & 3 & - & - \\
$\mathrm{MB}$ & 0.128 & 5.0 & 1000 & $10^{-6}$ & 3 & 1.192 & 5.59 \\
\hline
\end{tabular}

Table 3: Flow condition and sediment properties in the fixed bed ("FB") and mobile bed ("MB") sheet flow experiment of Revil-Baudard et al. (2015, 2016).

We first carried out a numerical simulation of the case FB to establish the accuracy of the present numerical model on hydrodynamics before presenting more complicated mobile bed sheet flow model validation. To simulate the flow over fixed rough bottom, a single layer of particles are fixed above the bottom boundary in the DEM (i.e., the particle velocities are zero and their positions are fixed). The rough wall function (Eqn. 34) is used with a bed roughness $k_{s}=2.5 d$ to estimate the $\omega_{b e d}$ in the turbulence closure. In the experiment of Revil-Baudard et al. (2016), the flow depth above the fixed particles is about $h=0.105 \mathrm{~m}$. The vertical domain length is chosen to be $L_{z}=h+d=0.108 \mathrm{~m}$ with a uniform grid size of $\Delta_{z}=0.25 \mathrm{~mm}$. Therefore, the fixed bed layer is resolved by the first 12 grid points above the bottom. The measured bottom frictional velocity in the experiment is $u_{*}=0.052 \mathrm{~m} / \mathrm{s}$. To match the bottom shear stress, the flow driving force is prescribed as $f_{x}=25.8 \mathrm{~Pa} / \mathrm{m}$. The model results of the fluid velocity, Reynolds shear stress and the TKE profiles are compared with the measured data in Fig. 4, where the fixed particle layer 

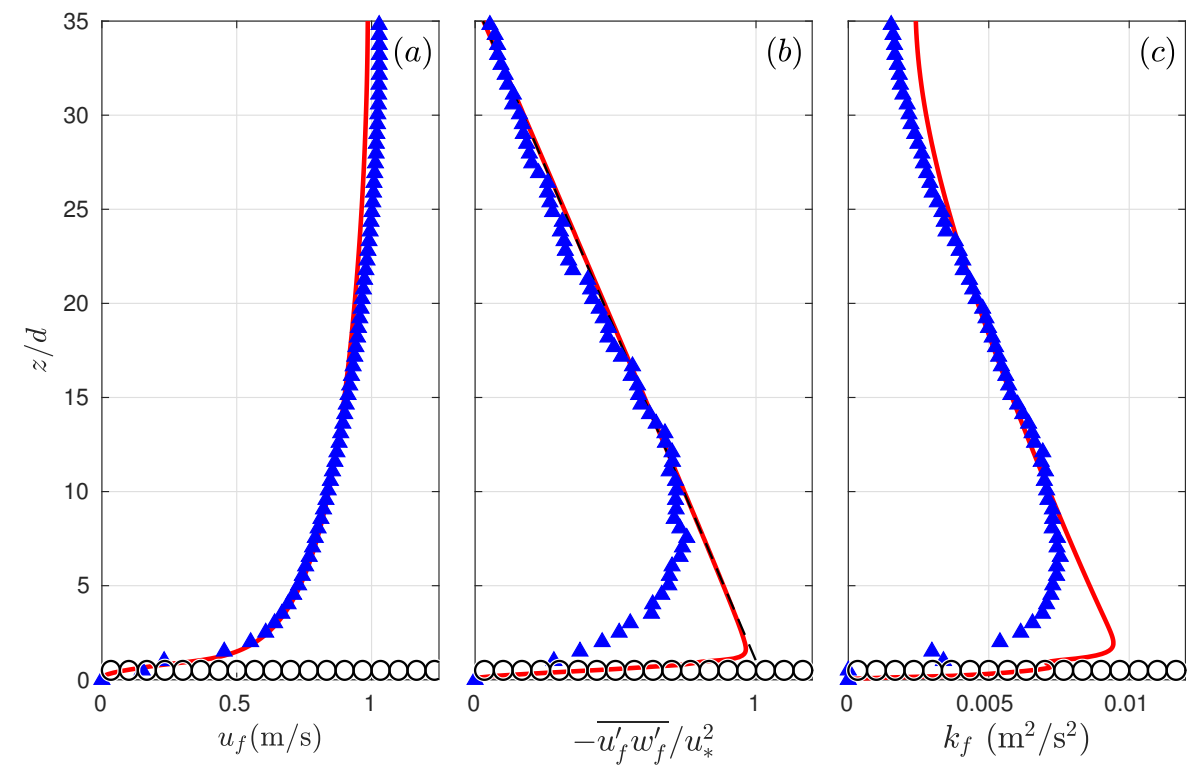

Figure 4: The comparison of (a) velocity profile, (b) normalized Reynolds shear stress and (c) TKE profile between numerical results (solid curves) and measured data (filled triangle symbols) for the fixed bed case ('FB') in the experiment of Revil-Baudard et al. (2016); In all panels, the fixed particle layer is denoted as circle symbols. In panel (b), the dashed curve is denotes a linear profile of the total shear stress.

is also denoted as circle symbols. Due to the drag force from the fixed particles above the bottom, the velocity profile drops to zero within the fixed bed layer, and good agreements in the streamwise velocity profiles are obtained with the measured data. The modeled Reynolds shear stress profile captures the linear decaying shape (dashed curve) and it matches the experimental data reasonably well. In particular, the Reynolds-averaged closure provides a good prediction of the TKE magnitude throughout most of the water column. The good agreement with the FB case confirms that the turbulence closure works well for the steady flow over a rough fixed sediment bed.

The mobile bed sheet flow (see case MB in Table 3) is then studied with a thick layer of particles at the bottom of the domain. To prepare the sediment bed, the particle velocities are initialized to be zero, and 43929 particles are well mixed in the whole domain. Due to the gravitational settling, the particles settle down to the bottom until their kinematic energies are minimized. After this initialization step, the initial bed level locates at 
$z=0.045 \mathrm{~m}$ above the bottom of the domain. Due to the sediment suspension, the final bed depth at the quasi-steady state will be smaller. Through a preliminary test, we determined that the total vertical domain height should be $L_{z}=0.168 \mathrm{~m}$ so that the final flow depth of $h=0.128 \mathrm{~m}$ (sediment bed location becomes $z_{b}=0.04 \mathrm{~m}$ ) can be obtained after the flow reaches the steady state. The vertical domain is discretized into 168 grid cells with a uniform grid size $\Delta_{z}=0.001 \mathrm{~m}$. The streamwise and spanwise domain lengths are $L_{x}=0.144 \mathrm{~m}$ and $L_{y}=0.072 \mathrm{~m}$. In these two horizontal directions, only one CFD grid cell is used in each direction. To confirm the model domain size is adequate, we carried out a comparative case by reducing the streamwise domain length by half $\left(L_{x}=0.072 \mathrm{~m}\right)$, and the model results on mean flow quantities show good convergence. The same coefficient $C_{0}=3$ calibrated for dilute suspension (see Section 3.1) is used here for the sheet flow simulation using the LRN $k-\omega$ model. The snapshot of the horizontal fluid velocity profile and sediment particle distribution after the flow reaches the statistically steady state is shown in Fig. 5. Although the flow is solved using a Reynolds-averaged turbulence closure, the stochastic motions of the sediment particles are captured by the eddy interaction model and particle collisions. As a result of the eddy interaction model, the sediment particles are suspended away from the bed via turbulent suspension.

The numerical results of the mean velocity profile, normalized concentration profile, sediment fluxes $\left(Q_{s}=\phi u_{s}\right)$ and TKE profiles are compared with the measured data in Fig. 6. To reduce the fluctuations due to stochastic motion of particles, time-averaging with a 10 second window is applied to calculate the mean flow quantity after the flow reaches steady state. In panel (a), we observe that the modeled fluid velocity profile is similar to the measured data in the upper water column $\left(\left(z-z_{b}\right)>7 d\right)$ when sediment concentration is very dilute. In the region of intermediate sediment concentration, $\left(0<\left(z-z_{b}\right) / d<7\right)$, sediment velocity is slightly smaller than the fluid velocity and agrees with measured velocity profile. This lag in sediment phase velocity is consistent with many particle-laden flow observations (e.g., Muste et al., 2005; Pal et al., 2016). The modeled velocity profiles without the eddy interaction model are similar and hence they are not shown here for 


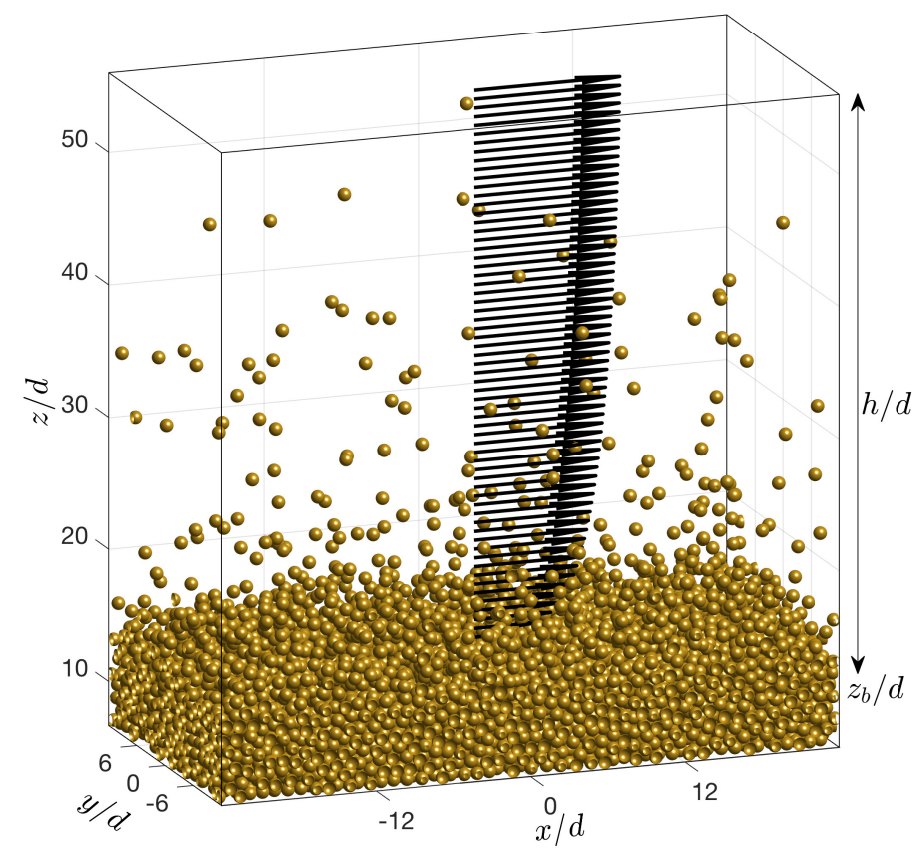

Figure 5: A snapshot of flow velocity field (arrows) and sediment particles (assumed to be spherical) for the entire computation domain along with the definition of coordinate system. The initial bed depth is denote as $z_{b}$, and the water depth is denoted as $h$.

brevity. Very near the bed $\left(\left(z-z_{b}\right) \leq 3 d\right)$, the model over-predicts the velocity gradient, while the measured data shows a milder increase of velocity away from the bottom in the range of $0<\left(z-z_{b}\right)<7 d$. As a result, the numerical model under-predicts the shear layer thickness above the bed. According to Revil-Baudard et al. (2015), the large nearbed shear layer observed in the experiment may be related to the nearbed intermittencies. Even though the EIM is used for the turbulence-sediment interaction, the stochastic model is still too simple to model the bed intermittency, and a turbulence-resolving simulation approach may be necessary for such feature (Cheng et al., 2017b).

The sediment concentration profiles normalized by the maximum sediment concentration $\phi_{\max }$ are compared in Fig. 6b. It shall be noted that our numerical model predicts that the maximum sediment concentration is $\phi_{\max } \approx 0.635$, while the measured data gives $\phi_{\max }=0.55$. The discrepancy in the maximum packing concentration is probably related to the non-spherical particle shape used in the experiments. From the normalized 

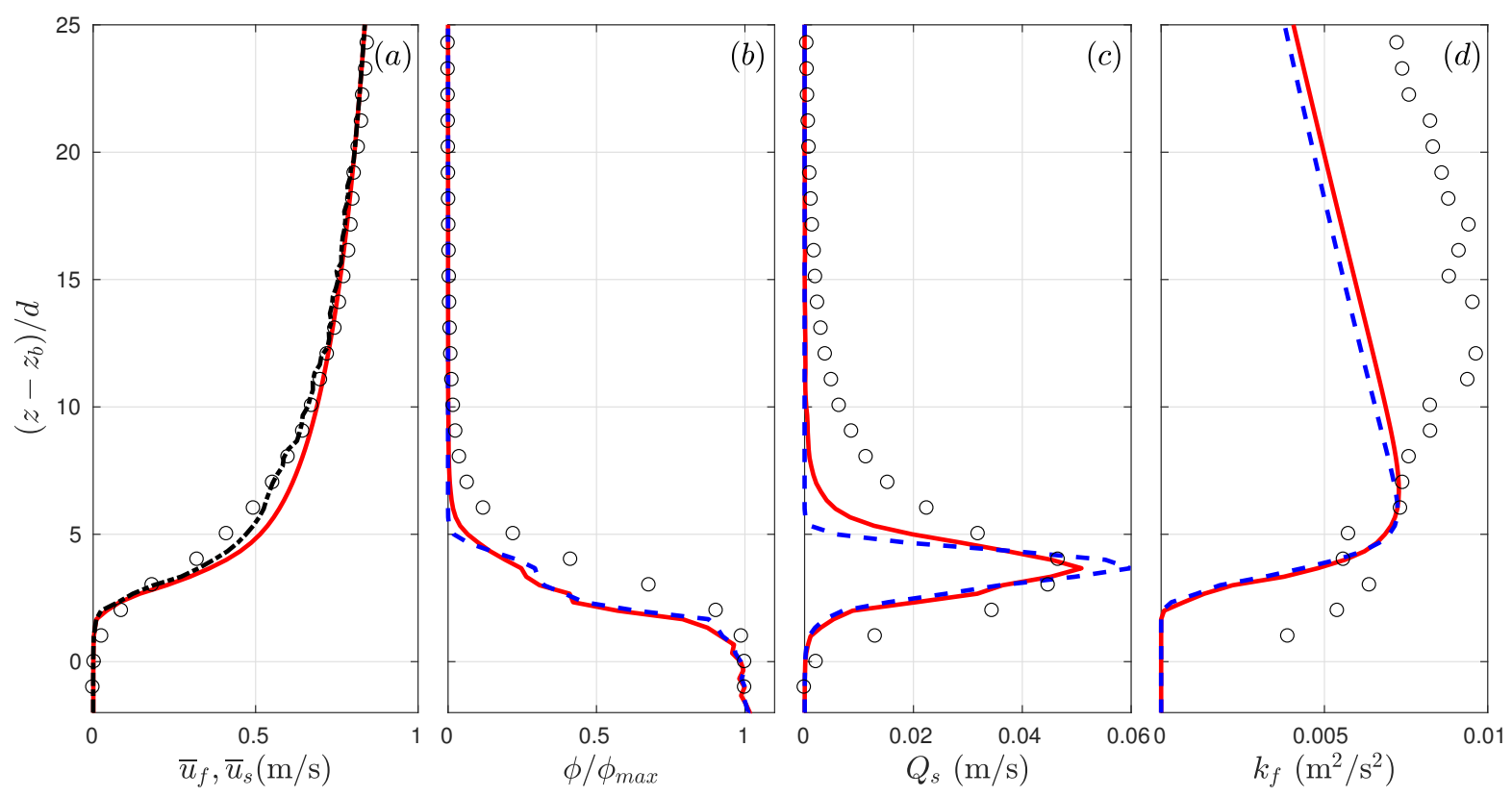

Figure 6: The comparison of (a) velocity profile, (b) normalized concentration profile and (c) sediment flux profile between numerical results and measured data with eddy interaction model (solid curve) and without eddy interaction model (dashed curve); In all panels, the circle symbols are measured data. In panel (a), solid curve denotes the fluid velocity, and dash-dot curve is the sediment velocity with EIM.

sediment concentration profiles, we can see that the modeled sediment concentration with EIM shows a more smooth vertical distribution and is more consistent with measured concentration profile. On the other hand, the concentration profile without the EIM indicates that a dense, thin transport layer is predicted between $3 d<\left(z-z_{b}\right)<5 d$. Consequently, excessive sediment accumulation occurs in this region, and sediment flux is over-predicted (see Fig. 6r). This feature is similar to typical bedload transport model results for much coarser particles or aeolian transport (Durán et al., 2012). Here, the 'shoulder-shape' concentration profile is clearly absent in the measured data and the model result with EIM shows a better agreement. At the higher Shields parameter and a fall parameter (ratio of settling velocity to friction velocity) around 1 or smaller, the suspended transport becomes non-negligible. This point will be discussed more extensively in Section 4.2. Comparisons presented here indicate that the EIM can effectively model the the turbulent diffusion of sediment concentration. Therefore, including the eddy-interaction model in 
Reynolds-averaged formulation is essential to accurately model sediment concentration. Although the concentration profile with $C_{0}=3$ captures the main features similar to the measured data, it is clear that the present model under-predicts the sediment suspension in the range of $5 d<\left(z-z_{b}\right)<10 d$, and hence the sediment flux is also under-predicted (see panel (c) in Fig. 6). While it is possible to further increase $C_{0}$ (increase turbulent suspension) to match the measured data better, it may not be physically valid. The TKE profiles are further compared with the measured data in Fig. 6d. Firstly, we can see that including/excluding the EIM has negligible impact on the modeled TKE profile, and both results show under-prediction of TKE away from the bed $\left(\left(z-z_{b}\right) / d>7\right)$ and very near the bed $\left(\left(z-z_{b}\right) / d<3\right)$. As presented in Fig. 44, the model predicts the TKE profile very well for fixed rough bed condition of similar bottom friction velocity. Intercomparison of the measured TKE between the "FB" and "MB" conditions indicate that turbulence is enhanced by about $40 \%$ away from the bed $\left(7<\left(z-z_{b}\right) / d<25\right)$ and a significant enhancement is also observed very near the bed $\left(\left(z-z_{b}\right) / d<3\right)$ in the mobile bed experiment. Revil-Baudard et al. (2016) attribute the enhancement of turbulence to near-bed intermittency. More recent Eulerian two-phase Large-eddy simulation study (Cheng et al., 2017b) further demonstrated that turbulence above the concentrated sheet layer $\left(\left(z-z_{b}\right) / d>7\right)$ can be enhanced through these frequent but intermittent sediment burst events. It is noted that the present turbulence-averaged model is not designed to capture these intermittent turbulent features.

In summary, including the eddy interaction model is required for the prediction of sediment concentration and sediment flux under sheet flow conditions. Although sediment concentration in the dilute region is under-predicted with $C_{0}=3$ in the EIM, the discrepancy is believed to be caused by under-prediction of turbulence due to intermittent turbulent features but not EIM itself. The sensitivity of the modeled suspended sediment concentration will be discussed in more details subsequently. 


\section{Discussion}

\subsection{Sensitivity of sediment diffusivity to the coefficient $C_{0}$}

As demonstrated in Section 3.1 for the channel flow with dilute sediment suspension, the sediment concentration profiles are sensitive to the coefficient $C_{0}$ in the eddy interaction model, and the suspended sediment concentration gradient increases with $C_{0}$ values. It is clear that the gradient of sediment concentration profile is related to the particle dispersion (or sediment diffusion). In this section, we further analyze the sensitivity of the suspended sediment concentrations and the sediment diffusivity to the coefficient $C_{0}$ under steady sheet flow conditions by varying $C_{0}=2,3,6$, and 8 .

The effect of $C_{0}$ values on the sediment concentration profile is illustrated in Fig. 7 . Similar to the Rouse profile in dilute particle-laden flows (Eqn. 38), the Rouse profile in the sheet flow can be determined as,

$$
\frac{\bar{\phi}}{\phi_{r}}=\left(\frac{z-z_{b}}{h+z_{b}-z} \frac{h+z_{b}-z_{r}}{z_{r}-z_{b}}\right)^{-R o}
$$

In practice, the Rouse profile is only applicable when the turbulent suspension is dominant while the particle-particle interactions are negligible. Therefore, the reference location $z_{r}$ is chosen to be the lowest elevation at which the Reynolds shear stress follows a linear profile. The shear stress profiles corresponding to different $C_{0}$ values are shown in Fig. 7a. The Reynolds shear stress profile is nearly unaffected by the $C_{0}$ value. Meanwhile, the Reynolds shear stress follows the linear distribution above $\left(z-z_{b}\right) / d=7.5$, and therefore it can be conjectured that the inter-granular stress becomes important below $\left(z-z_{b}\right) / d=7.5$ and a common reference location $z_{r}=z_{b}+7.5 d$ is chosen.

The normalized sediment concentration profiles are plotted in logarithmic scale in Fig. 7b, where the thick curves are numerical results, and thin dash-dot curves are the corresponding fitted Rouse profiles. The modeled sediment concentration profiles fit the Rouse profile well in the dilute region $\left(\left(z-z_{b}\right)>7 d\right)$ for all the $C_{0}$ values tested. However, different slopes of concentration profiles were observed by varying $C_{0}$ values. We quantify the slope of sediment concentration in logarithmic scale using the Rouse number $R o$ (see Eqn. 39p. For $C_{0}=2$, nearly no sediment is suspended above $\left(z-z_{b}\right) / d=15$ and the 

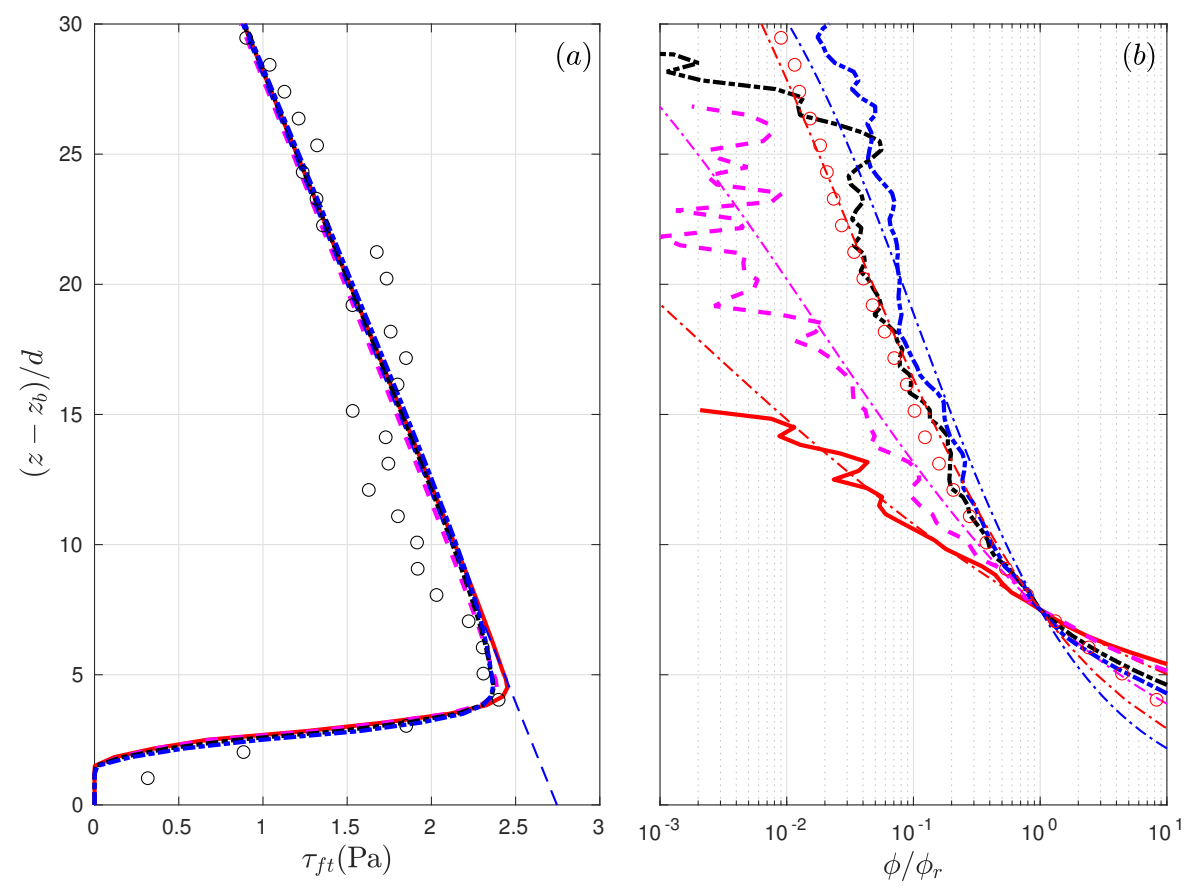

Figure 7: The comparison of (a) Reynolds shear stress profiles and (b) sediment concentration profiles plotted in semi-log scale for model result $\left(C_{0}=2\right.$, thick solid curve; $C_{0}=3$, thick magenta dashed curve; $C_{0}=6$, thick black dash-dot curve; $C_{0}=8$, thick blue dash-dot curve) and measured data (symbols). In panel (a), the thin dashed curve denotes a linear fit to the Reynolds shear stress profile. In panel (b), the thin dash-dot curves are the fitted Rouse profile with Rouse number $R o=4.78,2.98,2.42$ and 1.64 for the model results of $C_{0}=2,3,6$ and 8 , respectively. The value for the measured data is $R o=2.14$.

Rouse number $R o=4.78$ is large compared with the measured data $R o=2.14$. Using $C_{0}=3$, sediments are suspended much higher in the water column and the resulting $R o=2.98$ is significantly lower. Further increasing $C_{0}$ to 6 and 8 , the Rouse number reduced to 2.42 and 1.64. Although the model result using $C_{0}=6$ matches the measured sediment concentration profile, as discussed before, increasing $C_{0}$ may not be physically justified because the predicted suspended sediment concentration also depends on modeled turbulence quantities.

For given sediment properties and flow conditions, the Rouse number depends on the Schmidt number $S c$, which is defined as the ratio between the fluid turbulent eddy viscosity $\left(\nu_{f t}\right)$ and the sediment diffusivity $\left(\nu_{p}\right)$. In many Reynolds-averaged Eulerian 
simulations of sediment transport (e.g., Hsu et al., 2004; Revil-Baudard and Chauchat, 2013; Cheng et al., 2017a), the gradient transport assumption is adopted,

$$
\overline{w^{s^{\prime}} \phi^{\prime}}=-\nu_{p} \frac{\partial \bar{\phi}}{\partial z}
$$

where the sediment diffusivity is often parameterized by the turbulent eddy viscosity, $\nu_{p}=\nu_{f t} / S c$, with a constant Schmidt number (e.g., Hsu et al., 2003; Chen et al., 2011; Cheng et al., 2017a). Alternatively, the sediment diffusivity may be evaluated as $\nu_{p}=$ $-w_{s} \bar{\phi} /(\partial \bar{\phi} / \partial z)$ by considering the balance between the turbulent suspension flux and the settling flux, $\overline{w^{s^{\prime}} \phi^{\prime}}=w_{s} \bar{\phi}$. In the present model, the sediment motion is directly resolved by a Lagrangian approach, and the eddy interaction model is incorporated to simulate the sediment suspension by the flow turbulence. Therefore, it is interesting to evaluate the eddy interaction model in terms of the resulting sediment diffusivity and Schmidt number.

The vertical profiles of turbulent eddy viscosity and sediment diffusivity for $C_{0}=2$, 3, 6 and 8 are compared in Fig. $8(\mathrm{a})$ and (b). The turbulent eddy viscosity profiles obtained using different $C_{0}$ values are similar to each other and their vertical distributions are close to the measured data. However, the magnitude of the eddy viscosity is overpredicted compared with the measured data. Recall in Fig. 6(d) that the present model also under-predict TKE, we can conclude that the model may significantly under-predict $\omega$ due to inability to resolve intermittent turbulent motion and sediment burst. This may provide some useful insights to further improve the present $k-\omega$ model for twophase flow in the future. As shown in Fig. 8(b), the vertical profiles of the sediment diffusivities are sensitive to the $C_{0}$ values (see Fig. 8(b)), and the sediment diffusivity increases with the increasing values of $C_{0}$. Because discrepancies exist in both eddy viscosity and sediment diffusivity, the overall evaluation was also examined by the ratio of these two quantities, namely the Schmidt number. The resulting Schmidt numbers $\left(S c=\nu_{f t} / \nu_{p}\right)$ are presented in Fig. 8(c). We noticed that the predicted Schmidt number was more or less a constant in the suspension layer $\left(z-z_{b}\right)>6 d$ for all the runs regardless of $C_{0}$ values, and this feature is consistent with the measurement. With $C_{0}=3$ the resulting Schmidt number is around unity $(S c \approx 1)$, which is significantly larger than 

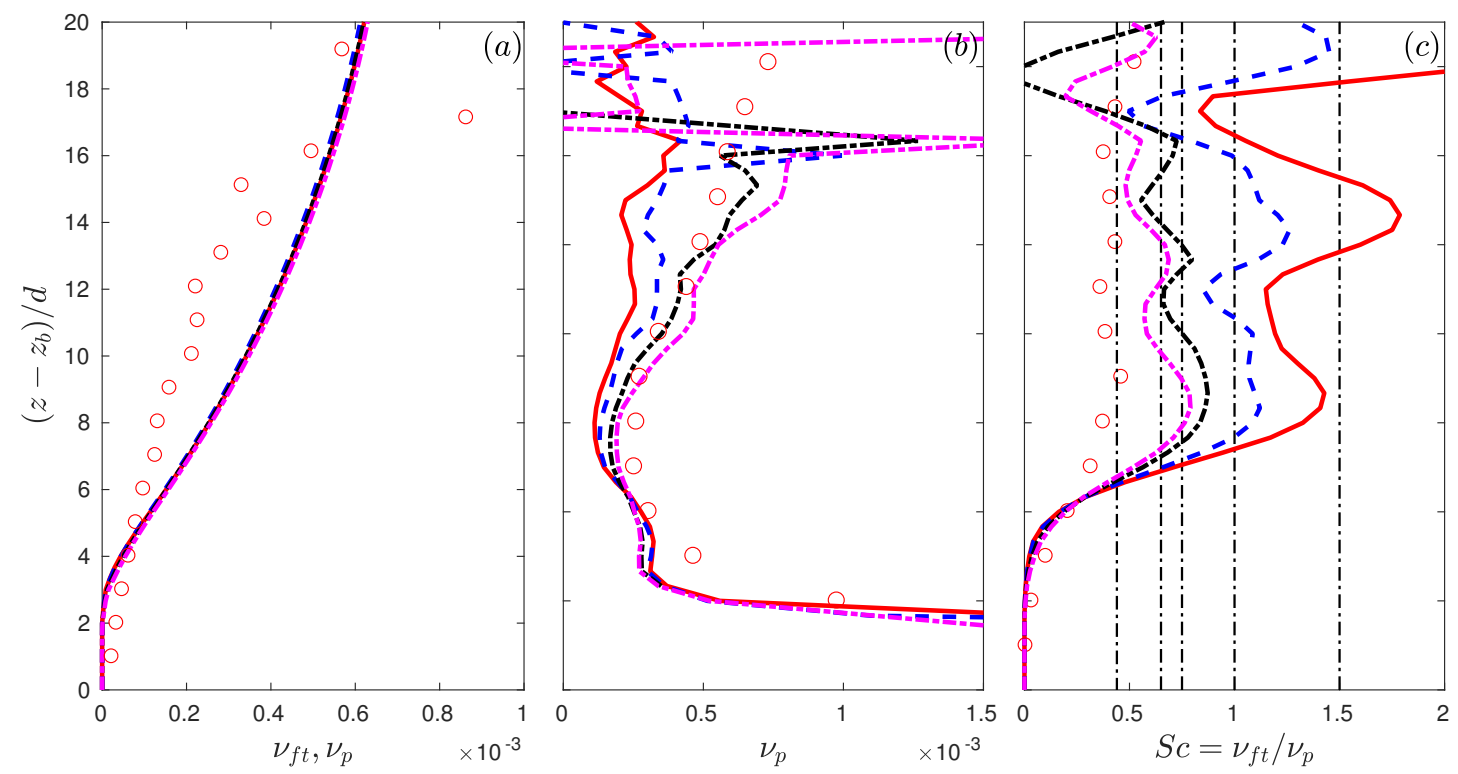

Figure 8: The vertical structure of turbulent eddy viscosity and sediment diffusivity are compared in panel (a) and (b), respectively. The corresponding vertical structure of Schmidt number $\left(S c=\nu_{f t} / \nu_{p}\right)$ is plotted in panel (c). In all three panels, model result with $C_{0}=2$ is denoted as thick solid curve, $C_{0}=3$ is denoted as thick dashed curve, $C_{0}=6$ is denoted as thick dash-dot curve and $C_{0}=8$ is denoted as magenta thick dash-dot curve. The symbols are the measured data. In panel (c), the thin dash-dot curves show the mean level of Schmidt number $(S c=0.44$ for measured data, $S c=1.5,1,0.75$ and 0.65 for model results with $C_{0}=2,3,6$ and 8, respectively).

the measured value $(S c \approx 0.44)$. The observed larger Schmidt number is consistent with the under-prediction of suspended sediment concentration and over-prediction of eddy viscosity discussed above. By increasing the value of $C_{0}$ to 6 and 8, the resulting Schmidt number decreased to $S c \approx 0.75$ and $S c \approx 0.65$, respectively. With this analysis, we can also conclude that simply increasing $C_{0}$ cannot reproduce the measured Schmidt because the eddy viscosity is over-predicted by the present two-phase flow $k-\omega$ model.

In summary, we showed that the discrepancies in the sediment diffusivity and Schmidt number could be due to the inability of the Reynolds-averaged model to capture the nearbed intermittencies as observed in the sheet flow experiment of Revil-Baudard et al. (2015). The nearbed intermittency enhances the turbulent intensities within the dense layer and upper water column. As a result, the present model under-predicted turbulent 
intensity in these regions, which can further cause the under-prediction of the suspended sediment concentration. To fully understand the dependence of Schmidt number on turbulent flow characteristics and sediment properties, a more sophisticated turbulenceresolving models may be needed. Secondly, several interphase momentum transfer forces such as the added mass and lift forces are neglected in the present study. It is expected that these interphase transfer forces are less important for heavy sand particles. However, they can become important for lightweight coarse particles (Jha and Bombardelli, 2010). Finally, we shall note that detailed experimental measurements on natural sand transport in sheet flow are needed to study the relevance of this nearbed intermittency of lightweight particles for the sand transport. More comprehensive investigations are warranted for future work.

\subsection{Transport rate and transport layer thickness}

The present model is applied to study the role of turbulent suspension (modeled by EIM) on sediment transport rate and transport layer thickness. In sediment transport applications, the sediment transport rate is often of high interest, as it is directly used in regional-scale models to study morphological evolutions (e.g., Lesser et al., 2004; Warner et al., 2008). Many steady flow experiments revealed that the dimensionless sediment transport rate can be parameterized by the non-dimensional bottom shear stress (e.g., Meyer-Peter and Muller, 1948; Nnadi and Wilson, 1992; Ribberink, 1998). The non-dimensional form of the bottom shear stress is called Shields parameter, $\theta=$ $\tau_{b} /\left[\left(\rho_{s}-\rho_{f}\right) g d\right]$. To evaluate the model capability to predict sediment transport rate, we carried out 14 cases with Shields parameter ranging from $\theta=0.3$ to 1.2 with/without EIM (see Table 4).

The resulting sediment concentration profiles and sediment flux profiles for three representative Shields parameters $(\theta=0.5,0.8$ and 1.2) are shown in Fig. 9, where panels (a, b) corresponds to the results with EIM, and panels $(c, d)$ corresponds to the results without EIM. As the shear stress exerted on the granular bed increases, the shear-induced dilation causes a larger erosion depth in the dense layer $\left(\bar{\phi} / \phi_{\max }>0.5\right.$ or $\left(z-z_{b}\right) / d<3$, see Fig. 9a and 9 ). This phenomenon is similar to the observations of Boyer et al. (2011) 


\begin{tabular}{cccccccc}
\hline$d(\mathrm{~mm})$ & $u_{*}(\mathrm{~cm} / \mathrm{s})$ & $\theta$ & $F=w_{s} / u_{*}$ & $\Psi_{b}$ & $\Psi_{t}$ & $\delta_{b} / d$ & $\delta_{t} / d$ \\
\hline 3 & 3.87 & 0.3 & 1.44 & 0.48 & 0.67 & 2.28 & 2.90 \\
3 & 4.47 & 0.4 & 1.25 & 0.93 & 1.16 & 2.90 & 4.15 \\
3 & 5.0 & 0.5 & 1.12 & 1.40 & 1.89 & 3.32 & 5.39 \\
3 & 5.48 & 0.6 & 1.02 & 1.81 & 2.85 & 3.94 & 6.43 \\
3 & 6.32 & 0.8 & 0.88 & 3.03 & 4.20 & 4.98 & 7.88 \\
3 & 7.07 & 1.0 & 0.79 & 5.01 & 7.39 & 6.01 & 10.16 \\
3 & 7.74 & 1.2 & 0.72 & 7.67 & 11.05 & 7.05 & 12.44 \\
\hline
\end{tabular}

Table 4: A summary of the numerical experiments to study the effect of EIM on the sediment transport rate and transport layer thickness at various Shields parameters. The transport rate and transport layer thickness with EIM are denoted as $\Psi_{t}$ and $\delta_{t}$, respectively, while the results without EIM are denoted as $\Psi_{b}$ and $\delta_{b}$, respectively.

for dense immersed granular flows, and it occurs regardless of the EIM. As a result of the shear-induced dilation, more sediments are eroded as the Shields parameter increases (the vertical location corresponding to $\bar{\phi} / \phi_{\max }=0.5$ is lower as $\theta$ increases). Between $3.5<\left(z-z_{b}\right) / d<10$, the turbulent suspension mechanism is missing without EIM, thus a steep concentration gradient is obtained in each case in Fig. 9(c). As a consequence of the much rapid decrease of sediment concentration below $\bar{\phi} / \phi_{\max }=0.3$, the sediment transport flux occurs mostly in the relatively dense layer (see Fig 9d, e.g., sediment flux is nearly zero for $\left(z-z_{b}\right) / d>8$ for the case with the highest Shields parameter). On the other hand, when EIM is incorporated to model turbulent suspension, sediments are suspended further away from the bed. The sediment transport flux in the relatively dilute layer $\left(\bar{\phi} / \phi_{\max }<0.3\right)$ is significantly larger, and the total flux is expected to be larger compared with the cases without EIM (see Fig. 9b).

The sediment transport rate can be obtained by integrating the sediment transport flux $\left(Q_{s}\right)$ over the entire vertical domain, and the dimensionless sediment transport rate 

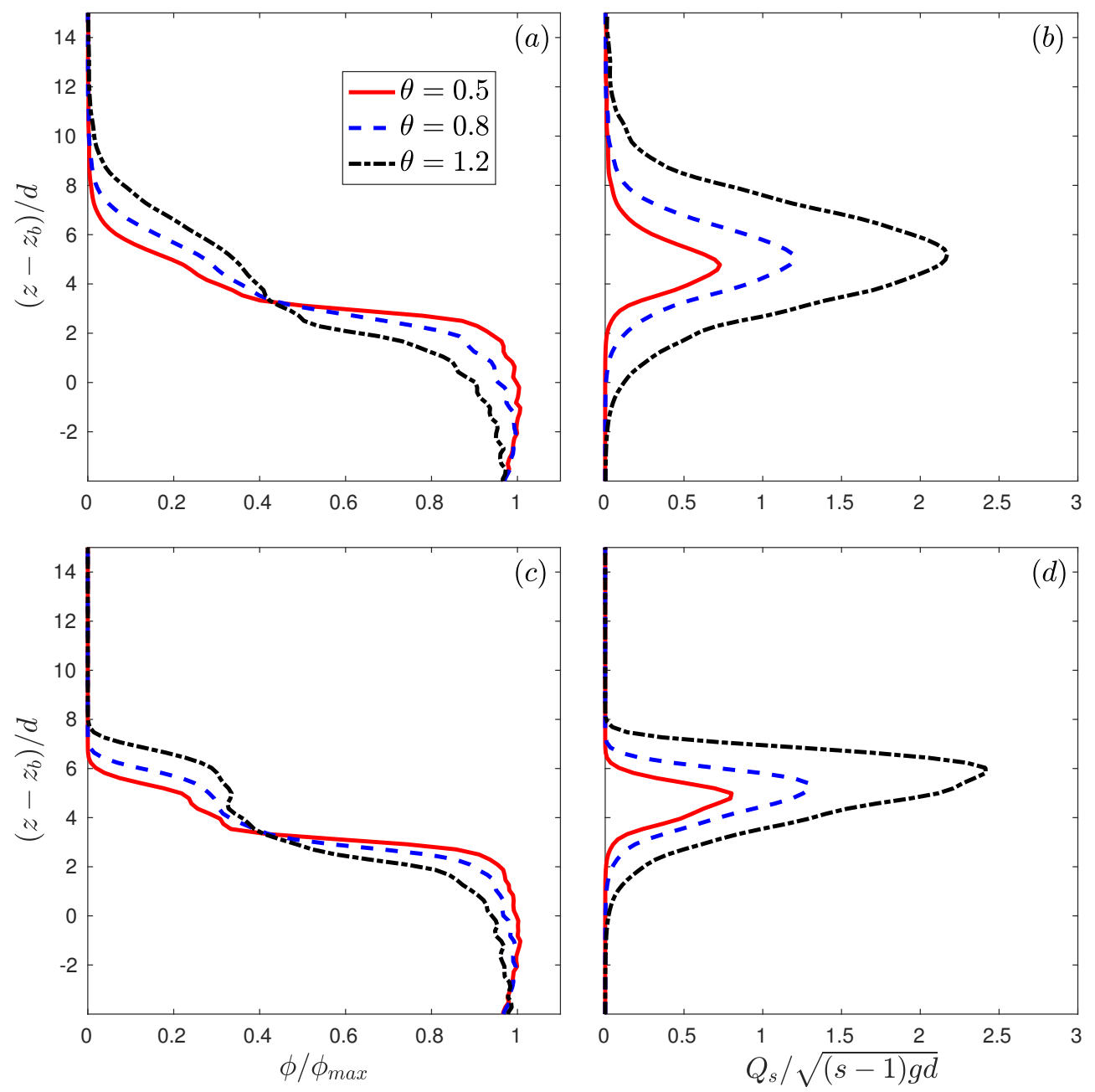

Figure 9: The sediment concentration profile and transport flux profile at three different Shields parameter, $\theta=0.5$ (solid curve), $\theta=0.8$ (dashed curve) and $\theta=1.2$ (dash-dot curve). Panel (a) and (b) corresponds to the result with eddy interaction model, while panel (c) and (d) are the results without eddy interaction model. The sediment concentration is normalized by the maximum sediment concentration $\phi_{\max }=0.635$, and the transport flux is normalized by $\sqrt{(s-1) g d}$.

can be computed as (Durán et al., 2012),

$$
\Psi=\frac{\sum_{i=1}^{N} \mathbf{v}_{i} V_{i} /\left(L_{x} L_{y}\right)}{\sqrt{(s-1) g d^{3}}} .
$$

In this study, the sediment transport rate obtained with EIM is denoted as $\Psi_{t}$, while the transport rate without EIM is denoted as $\Psi_{b}$. According to the previous experimental results on the sediment transport rate, a general form of power law relationships between 
the dimensionless sediment transport rate and the excess Shields parameter $\left(\theta-\theta_{c}\right)$ can be written as,

$$
\Psi=M_{0}\left(\theta-\theta_{c}\right)^{N_{0}},
$$

Where a typical critical Shields parameter $\theta_{c}=0.05$ is used, several different values of the coefficient $M_{0}$ and $N_{0}$ were proposed from various experimental results. On the basis of the flume experiments for rather coarse sand $(d>3 \mathrm{~mm})$ at low Shields parameter $(\theta<0.2)$, Meyer-Peter and Muller (1948) proposed that $M_{0}=5.7$ and $N_{0}=1.5$. This is the well-known power law where the transport rate is proportional to the $3 / 2$ power of the excess Shields parameter $\left(\theta-\theta_{c}\right)$. Based on the duct flow experiment with a smaller grain size $(d=0.7 \mathrm{~mm})$ at higher Shields parameters $(\theta>1)$, Nnadi and Wilson (1992) suggested that the coefficient $M_{0}$ should be increased to $M_{0}=12$. More recent study by Ribberink (1998) found that the power 3/2 should be increased to about 1.67 as the suspended load becomes important when the Shields parameter becomes larger.

The numerical results of the dimensionless sediment transport rates as a function of the Shields parameters are plotted in Fig. 10. Clearly, the sediment transport rates predicted with EIM (circle symbols) and without EIM (triangle symbols) increase rapidly when the Shields parameter increases, and this trend follows the empirical power law (Eqn. 42 very well. The dash-dot curve in Fig. 10 shows the power law with $N_{0}=1.5$ (Meyer-Peter and Muller, 1948) and the resulting best fit is $M_{0}=8.1$. However, the fitted curve with a power of $N_{0}=1.5$ over-predicts the sediment transport rate for lower Shields parameters $(\theta<1)$, while the transport rate in the higher Shields parameter range is under-predicted. On the other hand, the best fit of the power law for the present model results gives $M_{0}=8.27$ and $N_{0}=2.0$, which is consistent the the values reported by Ribberink (1998), $M_{0}=10.4$ and $N_{0}=1.67$. In addition, the transport rate without EIM is also compared with that of EIM. It is evident that the transport rate without EIM is generally smaller, and the discrepancy increases as the Shields parameter increases. If we further fit the transport rate obtained without EIM into the power law formula, we obtain that $M_{0}=5.5$ and $N_{0}=2.0$. It is interesting to note that although the proportionality constant $M_{0}$ is much lower than that of EIM, the power $N_{0}$ remains the same. 

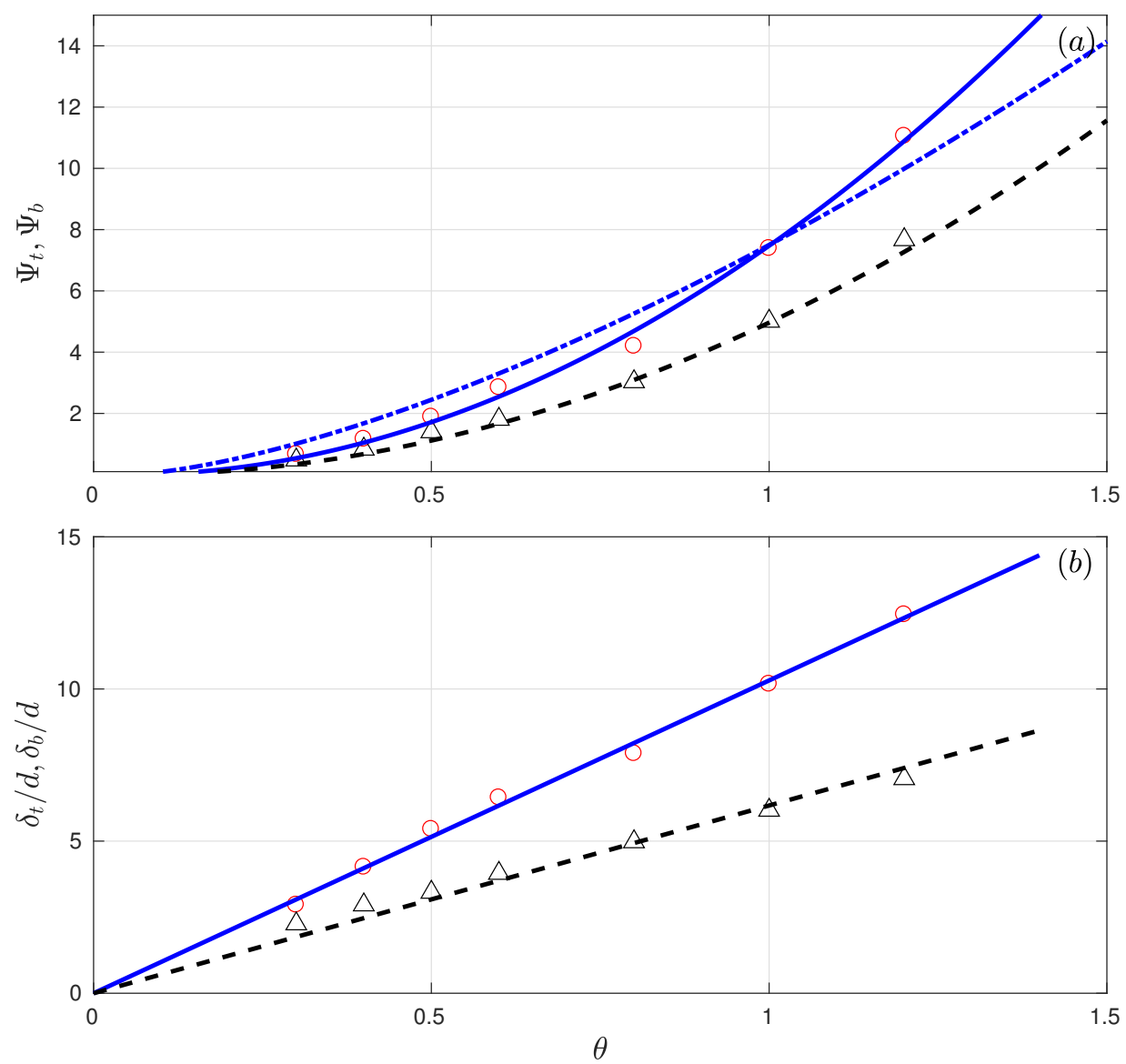

Figure 10: The nondimensional transport rate (panel a) and transport layer thickness (panel b) as a function of Shields parameter $\theta$. The circled symbols are model results with eddy interaction model (denoted as $\Psi_{t}$ ). To contrast the effect of EIM, the model results without EIM $\left(\Psi_{b}\right)$ are denoted as triangle symbols. The solid curve shows the empirical formulation of Eqn. (42) with $\theta_{c}=0.05, M_{0}=8.27$ and $N_{0}=1.97$, while the dash-dot curve corresponds to $\theta_{c}=0.05, M_{0}=8.1$ and $N_{0}=1.5$. The best fit to the transport rate without EIM is Eqn. (42) with $\theta_{c}=0.05, M_{0}=5.5$ and $N_{0}=2.0$. In panel (b), the solid curve is the linear fit transport layer thickness with EIM, while the dashed curve is for the cases without EIM.

As shown in Figure 9 (b) and (d), the sediment horizontal flux mainly occurs within a thick layer of about $10 \sim 15$ grain diameters above the bed. In sheet flow applications, the transport layer thickness is another quantity of interest, because this is where a large portion of transport takes place. For example, Wilson (1987) argues that mobile beds at high shear stresses can neither be considered as a rough or smooth fixed wall 
but they obey their own friction law with a frictional length scale proportional to the thickness of the major transport layer. Wilson (1987) defined the major transport layer thickness as the distance of the lowest mobile bed layer $\left(u_{s}<1 \mathrm{~mm} / \mathrm{s}\right)$ and the sediment concentration $\bar{\phi}=8 \%$. However, we noticed that using the $8 \%$ threshold may neglect too much transport for the present analysis and a lower threshold may be more appropriate. Here, we define the transport layer directly from the sediment flux profile, where the dimensionless sediment flux is larger than a small threshold: $Q_{s} / \sqrt{(s-1) g d}>0.05$. The resulting transport layer thickness with $\operatorname{EIM}\left(\delta_{t}\right)$ and without $\operatorname{EIM}\left(\delta_{b}\right)$ are compared in Fig. 10p. It is evident that the transport layer thickness increases with the Shields parameter. According to the experimental observations (e.g., Wilson, 1987; Sumer et al. 1996), the transport layer thickness is nearly proportional to the grain diameter and Shields parameter. As shown in Fig. 10p, we can see that a linear relationship can be found regardless of whether EIM is adopted or not, even though the proportionality coefficients are quite different. Without EIM, the transport layer thickness can be well described as $\delta_{b} / d=6.18 \theta$. However, the transport layer thickness with EIM is much larger, $\delta_{s} / d=10.28 \theta$ with the proportional coefficient very close to the value 10 as suggested by Wilson (1987).

According to Bagnold (1966), the particle suspension occurs when the dominant vertical velocity of the turbulent eddies exceeds the particle settling velocity. Assuming that the vertical velocity fluctuation can be quantified by the vertical turbulent velocity fluctuation, we can assume that the turbulent suspension is important if $w_{r m s}^{\prime}>w_{s}$. In the present model, an isotropic turbulence is assumed, such that the vertical turbulence intensity is approximated as, $w_{r m s}^{\prime} \approx \sqrt{2 k / 3}$. Nezu .1993$)$ suggested that the maximum TKE can be estimated as $4.78 u_{*}^{2}$ for turbulent flow over smooth bed. In the present sheet sediment transport with coarse light particles, the maximum TKE can be reasonably represented by $3 u_{*}^{2}$ (see Fig. 6 d), thus the turbulent suspension can be initiated when the shear velocity satisfies, $w_{s} / u_{*}<\sqrt{2}$. This is similar to the discussion of van Rijn (1984b) and Sumer et al. (1996), where they suggested that the relative importance between suspended load and bedload sediment transport can be categorized by the fall parameter, 
$F=w_{s} / u_{*}$. As summarized in Table 4, the fall parameter varies from 1.44 to 0.72 as the shear velocity increases from 3.87 to $7.74 \mathrm{~cm} / \mathrm{s}$. From the previous discussion on the sediment transport rate and transport layer thickness, it is found that the difference of the transport rate between the results with and without EIM is negligible when the Shields parameter is smaller than 0.5 (fall parameter $F \geq 1.25$ ). However, when the Shields parameter is larger than 0.5 (or $F<1.25$ ), the difference becomes noticeable.

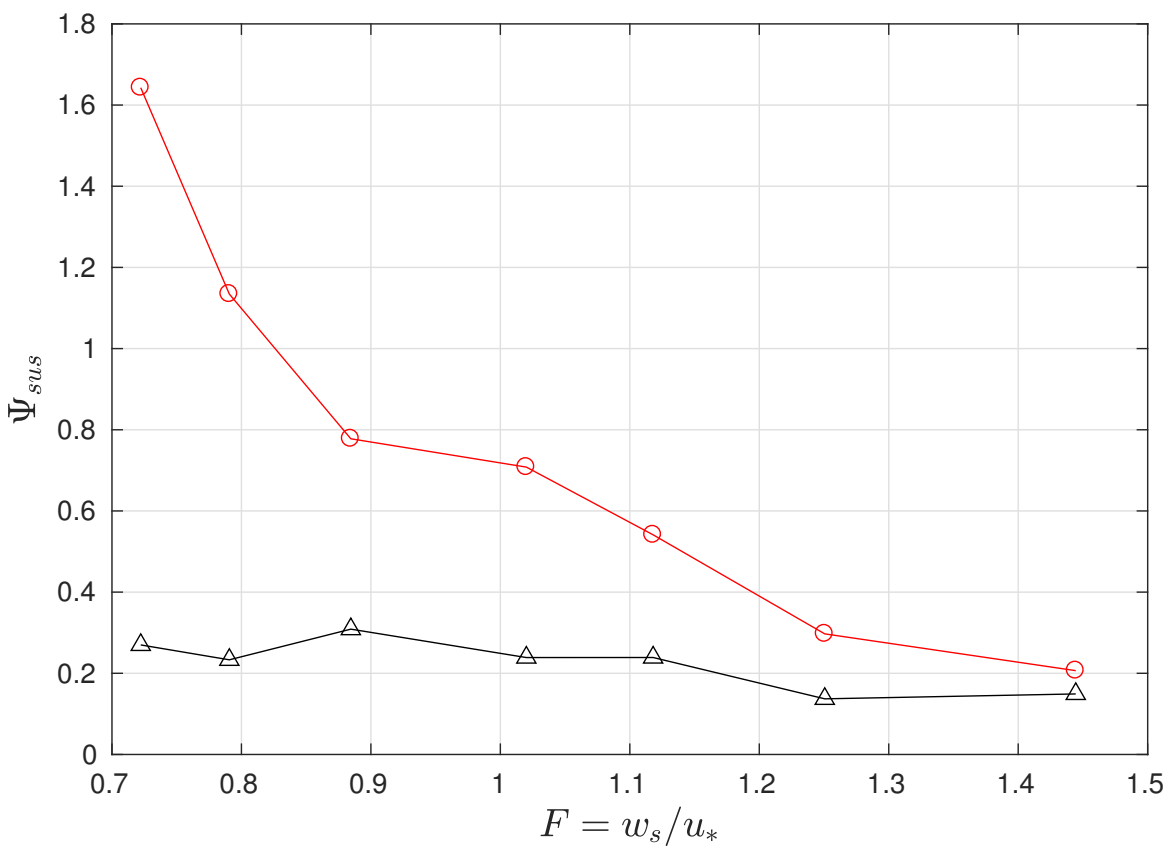

Figure 11: Nondimensional suspended sediment transport rate $\Psi_{\text {sus }}$ in the dilute region $(\phi<0.08)$ as a function of the fall parameter $F=w_{s} / u_{*}$. The circled symbols are model results with eddy interaction model, while the triangle symbol denotes the transport rate obtained without EIM.

To carry out more quantitative analysis, we consider that the turbulent suspension is most significant for the suspended load, which mainly occurs in the region of $\phi<0.08$. The non-dimensional suspended load sediment transport rate can be defined as,

$$
\Psi_{\text {sus }}=\frac{1}{\sqrt{(s-1) g d^{3}}} \int_{z(\phi=0.08)}^{L z} \phi u_{s} d z .
$$

To illustrate the importance of EIM on the prediction of suspended sediment flux, the suspended load with/without EIM are compared in Fig. 11 for a range of fall parameters. As the fall parameter increases, the sediment particles are less likely to be suspended 
by the turbulent eddies, thus the suspended sediment transport reduces rapidly. This is confirmed by the results of EIM, where the non-dimensional suspended sediment transport rate is reduced from 1.4 to 0.2 when the fall parameter increases from 0.72 to 1.44 . However, we can see that the suspended load predicted without EIM is quite small (around 0.2 ) and more or less a constant independent of the fall parameter. This indicates that the EIM is essential to capture the suspended sediment flux. For $F<1.25$, suspended load flux can be significantly under-predicted and EIM should be included in the EulerLagrange model for steady sheet flows.

\section{Conclusion}

In this paper, a Reynolds-averaged Euler-Lagrange sediment transport model was developed and applied to steady sheet flow, where the inter-granular interaction is directly resolved and the turbulent suspension of particles is modeled using an eddy interaction model. A LRN $k-\omega$ model extended for two-phase flow is implemented for the flow turbulence, which also provides the required turbulence statistics for the eddy-interaction model. The eddy interaction model was first calibrated using the dilute suspension experiments of Kiger and Pan (2002) and Muste et al. (2005). While the model is able to predict the measured flow velocity and turbulence kinetic energy very well, the model results are found to be sensitive to the coefficient $C_{0}$ associated with the eddy-particle interaction time (see Eqn. 36), and a value of $C_{0} \approx 3$ is calibrated to match the measured concentration profile in the dilute particle-laden flow.

After calibrating the eddy-interaction model for dilute suspension, an application of CFDEM-EIM to steady sheet flow was carried out by simulating the laboratory experiment of Revil-Baudard et al. (2015) with $C_{0}=3$. Although good agreements for flow velocity, turbulence kinetic energy, sediment concentration and sediment flux profiles are obtained for most of the sheet flow layer, the model clearly under-predicts turbulence and suspended sediment concentration in the dilute region. The under-predicted suspended sediment concentration is quantified by sediment diffusivity and we found that the sediment diffusivity decreases as the coefficient $C_{0}$ increases, while the fluid turbulent eddy 
viscosity is not sensitive to $C_{0}$ values. As a result, the resulting Schmidt number (ratio of fluid eddy viscosity to the sediment diffusivity) reduces as $C_{0}$ increases. However, the Schmidt number cannot be reduced to the measured value of 0.44 unless an unrealistic large value of $C_{0}$ is used. Therefore, it is likely that the under-prediction of suspended sediment concentration in the dilute region is mainly due to under-prediction of turbulence kinetic energy above the major sheet flow layer. As the higher level of turbulence may be associated with intermittent sediment burst events especially pronounced for lightweight particles (Revil-Baudard et al., 2015), a turbulence-resolving approach for the present Euler-Lagrange model may be necessary. Meanwhile, as the model can reproduce the major features of sheet flow layer, a model investigation was carried out to investigate the role of EIM and the resulting turbulent suspension on sediment transport rate and transport layer thickness. Model results confirmed that the non-dimensional transport rate follows a power law with the Shields parameter consistent with empirical formulations. Significant under-prediction of sediment transport rate were obtained without EIM due to lack of turbulent suspension, and the discrepancy between the result of EIM and without EIM is more pronounced when the fall parameter is lower than 1.25 (relatively smaller setting velocity or larger bottom friction velocity). Further analysis on transport layer thickness suggests that only when EIM is incorporated, the model is able to reproduce the well-known formula suggested by Wilson (1987).

Future improvements of the present CFDEM-EIM are suggested in the following aspects: First, the eddy interaction model is included only in the drag force, while the other interphase momentum transfer forces such as added mass and lift forces are ignored. However, their relative importance to the drag force in the eddy interaction model needs more investigations, especially for lightweight coarse particles. Secondly, even though the particles are tracked in a 3D domain with a Lagrangian approach, the fluid is solved only in a 1DV domain, and the flow is assumed to be homogeneous in the streamwise and spanwise directions. This assumption is reasonable for typical sheet flow conditions. However, for flows over nonuniform bathymetry or bedforms, this assumption is violated, and multidimensional simulations are needed for the fluid phase. Thirdly, the turbulence is assumed 
to be homogeneous and isotropic, thus the eddy interaction model may be too simple to reproduce the inhomogeneous features such as turbulent burst and preferential concentrations. Within the context of turbulence-averaged formulation, more sophisticated turbulence closure and eddy-interaction schemes can be pursued. Fourthly, it is noted that the model results are sensitive to the estimation of eddy life time, which is also highly variable based on the flow condition (Coimbra et al., 1998), and a more sophisticated turbulence model that directly resolves the eddy life time will be highly viable. Furthermore, to make good use of the coupled Euler-Lagrange scheme, CFDEM-EIM should be extensively applied to study the effects of grain size distribution and grain shape on sediment transport Calantoni et al., 2004; Calantoni and Thaxton, 2008; Fukuoka et al. 2014: Harada and Gotoh, 2008; Harada et al., 2015). Finally, the present study focused on developing a robust turbulence-averaged Euler-Lagrange model for various sediment transport applications. However, we also identified several outstanding issues in sheet flow sediment transport requiring further investigations, such as near bed intermittency and sediment diffusivity, which may require a turbulence-resolving simulation approach. Clearly, a fundamental understanding on many aspects of turbulence-particle interactions must be addressed by turbulence-resolving simulations and some encouraging works using the CFDEM framework have been reported (Schmeeckle, 2014; Sun and Xiao, 2016b).

\section{Acknowledgement}

Z. Cheng and T.-J. Hsu were supported by the U.S. Office of Naval Research (N0001416-1-2853) and National Science Foundation (OCE- 1537231). J. Chauchat was supported by the Région Rhones-Alpes (COOPERA project and Explora Pro grant) and the French national programme EC2CO-LEFE MODSED. J. Calantoni was supported under base funding to the U.S. Naval Research Laboratory from the U.S. Office of Naval Research. The authors would also like to acknowledge the support from the program on "FluidMediated Particle Transport in Geophysical Flows" at the Kavli Institute for Theoretical Physics, Santa Barbara, USA. The laboratory LEGI is part of the LabEx Tec 21 (Investissements d'Avenir - grant agreement nANR-11-LABX-0030) and Labex OSUG@2020 
(ANR10 LABX56). Numerical simulations were carried out on MILLS/FARBER at the University of Delaware. Simulations also leverage computing resource provided by Extreme Science and Engineering Discovery Environment (XSEDE) (TG-OCE100015). We are grateful to the developers involved in OpenFOAM, LIGGGHTS, LAMMPS and CFDEM, which are the foundation of the CFDEM-EIM presented in this paper. Dr. Xiao Yu, Mr. Samuel Bateman and Dr. Raphael Maurin provided many useful comments and insights to these open source codes and the EIM model.

\section{References}

J. Mossa, Sediment dynamics in the lowermost Mississippi River, Engineering Geology 45 (1) (1996) 457-479, ISSN 0013-7952.

M. A. Allison, C. R. Demas, B. A. Ebersole, B. A. Kleiss, C. D. Little, E. A. Meselhe, N. J. Powell, T. C. Pratt, B. M. Vosburg, A water and sediment budget for the lower Mississippi-Atchafalaya River in flood years 2008 2010: implications for sediment discharge to the oceans and coastal restoration in Louisiana, Journal of Hydrology 432 (2012) 84-97, ISSN 0022-1694.

M. Keshtpoor, J. A. Puleo, J. Gebert, N. G. Plant, Beach response to a fixed sand bypassing system, Coastal Engineering 73 (2013) 28-42, ISSN 0378-3839.

L. van Rijn, Sediment Transport, Part I: Bed Load Transport, Journal of Hydraulic Engineering 110 (10) (1984a) 1431-1456, ISSN 0733-9429.

L. van Rijn, Sediment Transport, Part II: Suspended Load Transport, Journal of Hydraulic Engineering 110 (11) (1984b) 1613-1641, ISSN 0733-9429.

T. O'Donoghue, S. Wright, Concentrations in oscillatory sheet flow for well sorted and graded sands, Coastal Engineering 50 (3) (2004) 117-138, ISSN 0378-3839.

T. Revil-Baudard, J. Chauchat, D. Hurther, P.-A. Barraud, Investigation of sheet-flow processes based on novel acoustic high-resolution velocity and concentration measurements, Journal of Fluid Mechanics 767 (2015) 1-30, ISSN 1469-7645. 
J. T. Jenkins, D. M. Hanes, Collisional sheet flows of sediment driven by a turbulent fluid, Journal of Fluid Mechanics 370 (1998) 29-52, ISSN 1469-7645.

S. Longo, Two-phase flow modeling of sediment motion in sheet-flows above plane beds, Journal of Hydraulic Engineering 131 (5) (2005) 366-379, ISSN 0733-9429.

T. Revil-Baudard, J. Chauchat, A two-phase model for sheet flow regime based on dense granular flow rheology, Journal of Geophysical Research: Oceans 118 (2) (2013) 619634, ISSN 2169-9291.

P. Dong, K. Zhang, Intense near-bed sediment motions in waves and currents, Coastal Engineering 45 (2) (2002) 75-87, ISSN 0378-3839.

T.-J. Hsu, J. T. Jenkins, P. L.-F. Liu, On two-phase sediment transport: sheet flow of massive particles, Proceedings of the Royal Society of London. Series A: Mathematical, Physical and Engineering Sciences 460 (2048) (2004) 2223-2250, ISSN 1364-5021.

L. Amoudry, T.-J. Hsu, P. L.-F. Liu, Two-phase model for sand transport in sheet flow regime, Journal of Geophysical Research: Oceans (1978-2012) 113 (C3), ISSN 21562202.

H. Liu, S. Sato, A two-phase flow model for asymmetric sheetflow conditions, Coastal Engineering 53 (10) (2006) 825-843, ISSN 0378-3839.

X. Chen, Y. Li, X. Niu, D. Chen, X. Yu, A two-phase approach to wave-induced sediment transport under sheet flow conditions, Coastal Engineering 58 (11) (2011) 1072-1088, ISSN 0378-3839.

Z. Cheng, T.-J. Hsu, J. Calantoni, SedFoam: A multi-dimensional Eulerian two-phase model for sediment transport and its application to momentary bed failure, Coastal Engineering 119 (2017a) 32-50, ISSN 0378-3839.

P. A. Cundall, O. D. Strack, A discrete numerical model for granular assemblies, Geotechnique 29 (1) (1979) 47-65, ISSN 0016-8505. 
R. Maurin, J. Chauchat, B. Chareyre, P. Frey, A minimal coupled fluid-discrete element model for bedload transport, Physics of Fluids (1994-present) 27 (11) (2015) 113302, ISSN 1070-6631.

R. Sun, H. Xiao, SediFoam: A general-purpose, open-source CFDDEM solver for particleladen flow with emphasis on sediment transport, Computers \& Geosciences 89 (2016a) 207-219, ISSN 0098-3004.

J. Calantoni, K. T. Holland, T. G. Drake, Modelling sheet-flow sediment transport in wave-bottom boundary layers using discrete-element modelling, Philosophical Transactions-Royal Society of London. Series A: Mathematical, Physical and Engineering Sciences 362 (2004) 1987-2002, ISSN 1364-503X.

E. Harada, H. Gotoh, Computational mechanics of vertical sorting of sediment in sheetflow regime by 3D granular material model, Coastal Engineering Journal 50 (01) (2008) 1945, ISSN 0578-5634.

S. Fukuoka, T. Fukuda, T. Uchida, Effects of sizes and shapes of gravel particles on sediment transports and bed variations in a numerical movable-bed channel, Advances in Water Resources 72 (2014) 84-96, ISSN 0309-1708.

J. R. Finn, M. Li, S. V. Apte, Particle based modelling and simulation of natural sand dynamics in the wave bottom boundary layer, Journal of Fluid Mechanics 796 (2016) 340-385, ISSN 0022-1120.

K. C. Wilson, Analysis of bed-load motion at high shear stress, Journal of Hydraulic Engineering 113 (1) (1987) 97-103, ISSN 0733-9429.

O. Durán, B. Andreotti, P. Claudin, Numerical simulation of turbulent sediment transport, from bed load to saltation, Physics of Fluids 24 (10) (2012) 103306, ISSN 10706631.

T. G. Drake, J. Calantoni, Discrete particle model for sheet flow sediment transport in 
the nearshore, Journal of Geophysical Research: Oceans 106 (C9) (2001) 19859-19868, ISSN 2156-2202.

R. Bagnold, An approachto the sedi ment transport problemfromgeneral physics, Tech. Rep., US Geological Survey Professional Paper, 1966.

B. M. Sumer, A. Kozakiewicz, J. Fredsoe, R. Deigaard, Velocity and concentration profiles in sheet-flow layer of movable bed, Journal of Hydraulic Engineering 122 (10) (1996) 549-558, ISSN 0733-9429.

G. I. Taylor, Diffusion by continuous movements, Proceedings of the London Mathematical Society 20 (1) (1922) 196-212.

C. Coimbra, J. Shirolkar, M. Q. McQuay, Modeling particle dispersion in a turbulent, multiphase mixing layer, Journal of Wind Engineering and Industrial Aerodynamics 73 (1) (1998) 79-97, ISSN 0167-6105.

H. Shi, X. Yu, An effective Euler-Lagrange model for suspended sediment transport by open channel flows, International Journal of Sediment Research 30 (4) (2015) 361-370, ISSN 1001-6279.

S. Balachandar, J. K. Eaton, Turbulent dispersed multiphase flow, Annual Review of Fluid Mechanics 42 (2010) 111-133, ISSN 0066-4189.

E. Matida, W. Finlay, C. Lange, B. Grgic, Improved numerical simulation of aerosol deposition in an idealized mouth-throat, Journal of Aerosol Science 35 (1) (2004) 1-19, ISSN 0021-8502.

D. Graham, On the inertia effect in eddy interaction models, International journal of multiphase flow 22 (1) (1996) 177-184, ISSN 0301-9322.

C. Goniva, C. Kloss, N. G. Deen, J. A. Kuipers, S. Pirker, Influence of rolling friction on single spout fluidized bed simulation, Particuology 10 (5) (2012) 582-591, ISSN 1674-2001. 
C. Kloss, C. Goniva, A. Hager, S. Amberger, S. Pirker, Models, algorithms and validation for opensource DEM and CFDDEM, Progress in Computational Fluid Dynamics, an International Journal 12 (2-3) (2012) 140-152, ISSN 1468-4349.

T. Revil-Baudard, J. Chauchat, D. Hurther, O. Eiff, Turbulence modifications induced by the bed mobility in intense sediment-laden flows 808 (2016) 469-484, ISSN 0022-1120.

S. Luding, Introduction to discrete element methods: basic of contact force models and how to perform the micro-macro transition to continuum theory, European Journal of Environmental and Civil Engineering 12 (7-8) (2008) 785-826, ISSN 1964-8189.

R. Mindlin, Compliance of elastic bodies in contact, Journal of Applied Mechanics 16 (1949) 259-268.

A. Di Renzo, F. P. Di Maio, An improved integral non-linear model for the contact of particles in distinct element simulations, Chemical engineering science 60 (5) (2005) 1303-1312, ISSN 0009-2509.

M. R. Maxey, J. J. Riley, Equation of motion for a small rigid sphere in a nonuniform flow, Physics of Fluids (1958-1988) 26 (4) (1983) 883-889.

Z. Zhou, S. Kuang, K. Chu, A. Yu, Discrete particle simulation of particlefluid flow: model formulations and their applicability, Journal of Fluid Mechanics 661 (2010) 482-510, ISSN 1469-7645.

A. Haider, O. Levenspiel, Drag coefficient and terminal velocity of spherical and nonspherical particles, Powder technology 58 (1) (1989) 63-70, ISSN 0032-5910.

R. Di Felice, The voidage function for fluid-particle interaction systems, International Journal of Multiphase Flow 20 (1) (1994) 153-159, ISSN 0301-9322.

J. A. Simeonov, S. Bateman, J. Calantoni, Filter width and uncertainty estimation in continuum modeling of particle phases, International Journal of Multiphase Flow 74 (2015) 79-83, ISSN 0301-9322. 
S. Pirker, D. Kahrimanovic, C. Goniva, Improving the applicability of discrete phase simulations by smoothening their exchange fields, Applied Mathematical Modelling 35 (5) (2011) 2479-2488, ISSN 0307-904X.

J. Capecelatro, O. Desjardins, An Euler-Lagrange strategy for simulating particle-laden flows, Journal of Computational Physics 238 (2013) 1-31, ISSN 0021-9991.

D. C. Wilcox, The remarkable ability of turbulence model equations to describe transition (1992) - .

L. O. Amoudry, Extension of $k-\omega$ turbulence closure to two-phase sediment transport modelling: Application to oscillatory sheet flows, Advances in Water Resources 72 (2014) 110-121, ISSN 0309-1708.

J. Chauchat, Z. Cheng, T. Nagel, C. Bonamy, T.-J. Hsu, SedFoam-2.0: a 3D two-phase flow numerical model for sediment transport, Geosci. Model Dev. Discuss. 2017 (2017) 1-42, ISSN 1991-962X.

D. Milojeviè, Lagrangian Stochastic-Deterministic (LSD) Predictions of Particle Dispersion in Turbulence, Part. Part. Syst. Charact. 7 (1-4) (1990) 181-190, ISSN 1521-4117.

K. Guizien, M. DohmenJanssen, G. Vittori, 1DV bottom boundary layer modeling under combined wave and current: Turbulent separation and phase lag effects, Journal of Geophysical Research: Oceans 108 (C1), ISSN 2156-2202.

W. Rodi, Examples of calculation methods for flow and mixing in stratified fluids, J. Geophys. Res. 92 (C5) (1987) 5305-5328, ISSN 2156-2202.

D. C. Wilcox, Comparison of two-equation turbulence models for boundary layers with pressure gradient, AIAA journal 31 (8) (1993) 1414-1421, ISSN 0001-1452.

M. Muste, K. Yu, I. Fujita, R. Ettema, Two-phase versus mixed-flow perspective on suspended sediment transport in turbulent channel flows, Water Resources Research 41 (10), ISSN 1944-7973. 
F. Menter, T. Esch, Elements of industrial heat transfer predictions, in: 16th Brazilian Congress of Mechanical Engineering (COBEM), vol. 109, 2001.

J. Bredberg, S. Peng, L. Davidson, On the Wall Boundary Condition for Computing Turbulent Heat Transfer With $k-\omega$ Models, ASME-PUBLICATIONS-HTD 366 (2000) 243-250, ISSN 0272-5673.

D. C. Wilcox, Reassessment of the scale-determining equation for advanced turbulence models, AIAA journal 26 (11) (1988) 1299-1310, ISSN 0001-1452.

D. R. Fuhrman, M. Dixen, N. G. Jacobsen, Physically-consistent wall boundary conditions for the k- turbulence model, Journal of Hydraulic Research 48 (6) (2010) 793-800, ISSN 0022-1686.

D. Graham, P. James, Turbulent dispersion of particles using eddy interaction models, International Journal of Multiphase Flow 22 (1) (1996) 157-175, ISSN 0301-9322.

G. Kallio, M. Reeks, A numerical simulation of particle deposition in turbulent boundary layers, International Journal of Multiphase Flow 15 (3) (1989) 433-446, ISSN 03019322.

V. Mehrotra, G. D. Silcox, P. J. Smith, Numerical simulation of turbulent particle dispersion using a Monte Carlo approach, in: Anon (Ed.), American Society of Mechanical Engineers, Fluids Engineering Division (Publication) FED Fairfield, NJ, United States: ASME., 1998.

A. Gosman, E. Loannides, Aspects of computer simulation of liquid-fueled combustors, Journal of Energy 7 (6) (1983) 482-490, ISSN 0146-0412.

H. Weller, Derivation, modelling and solution of the conditionally averaged two-phase flow equations, Tech. Rep., OpenCFD Ltd., 2002.

P. K. Sweby, High Resolution Schemes Using Flux Limiters for Hyperbolic Conservation Laws, SIAM Journal on Numerical Analysis 21 (5) (1984) 995-1011, ISSN 00361429. 
H. Rusche, Computational fluid dynamics of dispersed two-phase flows at high phase fractions, Ph.D. thesis, Imperial College London (University of London), 2002.

K. Kiger, C. Pan, Suspension and turbulence modification effects of solid particulates on a horizontal turbulent channel flow, J. Turbulence 3 (19) (2002) 1-17.

R. D. Moser, J. Kim, N. N. Mansour, Direct numerical simulation of turbulent channel flow up to Re $=590$, Physics of fluids 11 (4) (1999) 943-945, ISSN 1070-6631.

S. K. Jha, F. A. Bombardelli, Two-phase modeling of turbulence in dilute sediment-laden, open-channel flows, Environmental fluid mechanics 9 (2) (2009) 237-, ISSN 1567-7419.

V. Vanoni, Sedimentation Engineering, American Society of Civil Engineers, 2006.

D. Pal, S. K. Jha, K. Ghoshal, Velocity lag between particle and liquid in sediment-laden open channel turbulent flow, European Journal of Mechanics - B/Fluids 56 (2016) 130-142, ISSN 0997-7546.

Z. Cheng, T.-J. Hsu, J. Chauchat, An Eulerian two-phase model for steady sheet flow using large-eddy simulation methodology, Advances in Water Resources, under revision

T.-J. Hsu, J. T. Jenkins, P. L.-F. Liu, On two-phase sediment transport: Dilute flow, Journal of Geophysical Research: Oceans (1978-2012) 108 (C3), ISSN 2156-2202.

S. K. Jha, F. A. Bombardelli, Toward two-phase flow modeling of nondilute sediment transport in open channels, Journal of Geophysical Research: Earth Surface 115 (F03015), ISSN 2156-2202.

G. Lesser, J. Roelvink, J. van Kester, G. Stelling, Development and validation of a threedimensional morphological model, Coastal Engineering 51 (89) (2004) 883-915, ISSN 0378-3839. 
J. C. Warner, C. R. Sherwood, R. P. Signell, C. K. Harris, H. G. Arango, Development of a three-dimensional, regional, coupled wave, current, and sediment-transport model, Computers \& Geosciences 34 (10) (2008) 1284-1306, ISSN 0098-3004.

E. Meyer-Peter, R. Muller, Formulas for bed-load transport, in: Hydraulic Engineering Reports, IAHSR 2nd meeting, Stockholm, appendix 2, 1948.

F. N. Nnadi, K. C. Wilson, Motion of Contact-Load Particles at High Shear Stress, Journal of Hydraulic Engineering 118 (12) (1992) 1670-1684.

J. S. Ribberink, Bed-load transport for steady flows and unsteady oscillatory flows, Coastal Engineering 34 (12) (1998) 59-82, ISSN 0378-3839.

F. Boyer, E. Guazzelli, O. Pouliquen, Unifying Suspension and Granular Rheology, Phys. Rev. Lett. 107 (18) (2011) 188301.

I. Nezu, Turbulence in open-channel flows, A. A. Balkema, 1993.

J. Calantoni, C. S. Thaxton, Simple power law for transport ratio with bimodal distributions of coarse sediments under waves, Journal of Geophysical Research 113 (C3), ISSN 2156-2202.

E. Harada, H. Gotoh, N. Tsuruta, Vertical sorting process under oscillatory sheet flow condition by resolved discrete particle model, Journal of hydraulic research 53 (3) (2015) 332-350, ISSN 0022-1686.

M. W. Schmeeckle, Numerical simulation of turbulence and sediment transport of medium sand, Journal of Geophysical Research: Earth Surface 119 (6) (2014) 1240-1262, ISSN 2169-9011.

R. Sun, H. Xiao, CFD-DEM simulations of current-induced dune formation and morphological evolution, Advances in Water Resources 92 (2016b) 228-239, ISSN 0309-1708. 